Research Article

\title{
Hesitant Fuzzy Linguistic Hamy Mean Aggregation Operators and Their Application to Linguistic Multiple Attribute Decision-Making
}

\author{
Yuan Rong $\mathbb{D}^{1},{ }^{1}$ Zheng Pei $\mathbb{D}{ }^{1}{ }^{1}$ and Yi Liu $\mathbb{D D}^{2,3}$ \\ ${ }^{1}$ School of Science, XiHua University, Chengdu 610039, China \\ ${ }^{2}$ Data Recovery Key Laboratory of Sichuan Province, Neijiang Normal University, Neijiang 641000, Sichuan, China \\ ${ }^{3}$ Numerical Simulation Key Laboratory of Sichuan Province, Neijiang Noraml University, Neijiang 641000, Sichuan, China
}

Correspondence should be addressed to Zheng Pei; pqyz@263.net

Received 10 October 2019; Revised 29 December 2019; Accepted 16 January 2020; Published 19 February 2020

Academic Editor: Alessio Ishizaka

Copyright ( ) 2020 Yuan Rong et al. This is an open access article distributed under the Creative Commons Attribution License, which permits unrestricted use, distribution, and reproduction in any medium, provided the original work is properly cited.

\begin{abstract}
Linguistic aggregation operator is a paramount appliance to fix linguistic multiple attribute decision-making (MADM) issues. In the article, the Hamy mean (HM) operator is utilized to fuse hesitant fuzzy linguistic (HFL) information and several novel HFL aggregation operators including the hesitant fuzzy linguistic Hamy mean (HFLHM) operator, weighted hesitant fuzzy linguistic Hamy mean (WHFLHM) operator, hesitant fuzzy linguistic dual Hamy mean (HFLDHM) operator, and weighted hesitant fuzzy linguistic dual Hamy mean (WHFLDHM) operator are proposed. Besides, several paramount theorems and particular cases of these aggregation operators are investigated in detail, and then a novel MADM approach is presented by using the proposed aggregation operators. Ultimately, a practical example is utilized to manifest the effectiveness and practicability of the propounded method.
\end{abstract}

\section{Introduction}

The essence of MADM is the process of sorting a limited number of alternatives under given attributes and selecting the optimal one through the evaluation information provided by experts. It has been widely applied in multitudinous aspects [1-9]. Owing to the complicacy and nondeterminacy of the decision-making (DM) context, one of the most arduous challenges in course of MADM is how to represent the evaluation information of attributes in fuzzy and uncertain circumstances accurately. For handling this limitation, Zadeh [10] originally brought forward the notion of fuzzy set (FS), which can effectively depict vague and uncertain information through a membership degree. Thereafter, FS has been further investigated and acquired a large number of research achievements by multitude scholars in diverse aspects. Among them, the most important aspect is the extension of FS, for instance, intuitionistic FS [11], interval- valued intuitionistic FS [12], pythagorean FS [13], q-rung orthopair FS [14], and neutrosophic set [15] . Since then, a multitude of affluent research achievements were acquired on the basis of these extended FSs [16-22]. However, the aforementioned FSs have a common limitation which they only have a membership degree or no-membership degree to express preference information of decision makers (DMs). It cannot deal with several situations which DMs cannot ascertain a satisfied membership degree from a collection of membership degrees. Accordingly, for coping with this circumstance, Torra and Narukawa $[23,24]$ put forward the notion of hesitant FS (HFS), which enables the membership degree of an element belonging to a fixed objective to have multiple feasible values between 0 and 1 . Subsequently, a lot of plentiful research results including theory and methodology on HFS have been achieved. These achievements are summarized as the following aspects: (1) the foundation theory aspect: operations and algebraic structures $[25,26]$, 
information entropy measure [27, 28] distance measure, and similarly measure [29-31]; (2) the MADM approaches on HFS: Zhang and Wei [32] proposed the extension VIKOR approach to resolve the DM problem with hesitant fuzzy information. Zhang and $\mathrm{Xu}$ [33] propounded TODIM approach on the basis of a new measure function to deal with DM issues. Chen and $\mathrm{Xu}$ [34] extended the traditional ELECTRE II approach to handle hesitant fuzzy MADM problems; (3) the aggregation operator aspect: Xia and $\mathrm{Xu}$ [35] presented several aggregation operators for aggregating hesitant fuzzy information. He et al. [36] combined the power operator and Bonferroni mean (BM) operator to fuse hesitant fuzzy information, which can take into account the importance and inter-relationship of the aggregated data. Hong et al. [37] propounded the hesitant fuzzy dual Muirhead mean operators whose characteristic is that the correlation of attributes is taken into consideration.

Influenced by the complicacy and vagueness of information, DMs cannot provide their preference information in the form of a precise number in actual world. Especially, the common evaluation langue terms are "very good," "good," "bad," and "very bad." These linguistic terms are in line with DMs' assessment thinking for providing evaluation information to a particular project. Accordingly, the linguistic term set (LTS) is more convenient to express assessment information of alternatives than numerical number. Inspired by HFS, Rodriguez et al. [38] developed the hesitant fuzzy linguistic term set by combining the HFS and the LTS where DMs provide their evaluation information for a linguistic variable through some orderly and continuous linguistic terms. Although HFLTS can express assessment information qualitative, it cannot take into account the situation that the membership degree of a linguistic term belongs to a selected objective. To surmount this limitation, Lin et al. [39] introduced the notion of hesitant fuzzy linguistic set (HFLS), which allows several possible membership degrees to belong to a selected linguistic term, and the foundation element of HFLS is named HFLE. At the same time, several aggregation operators including the HFL geometric operator, HFL correlated operator, induced HFL operator, HFL prioritized operator, and HFL power operator are investigated. From the definition of HFLS, we can know that a HFLE includes two aspect information; on the one hand, the nature langue is used to express evaluation information through the linguistic term, and on the other hand, the hesitancy of DMs to a linguistic term is taken into consideration. Thereafter, Wang et al. [40] proposed the Hausdorff distance of HFL numbers and established novel HFL-TOPSIS and TODIM approach. Gou et al. [41] presented the HFL Bonferroni mean (HFLBM) operator and weighted HFLBM (WHFLBM) operator. It is obvious that the HFLS has stronger capacity to portray no-indeterminacy and vague information than HFS or LTS.

Aggregation function $[42,43]$ is one of the hot spots in the domain of the information process system, such as information fusion, pattern recognition, and decision support system. It is widely utilized to reduce the dimensions of assessment information during information aggregation procedure, such as triangular norms $[44,45]$, copulas [46] and arithmetic mean. It is of great importance in the process for collecting related assessment information from multiple evaluators in MADM issues. The main thought of the aggregation operator is to aggregate the evaluation information of alternatives under different attributes to get the total comprehensive evaluation information of alternatives. Traditional aggregation operators like weighted average, weighted geometric, and ordered weighted average (OWA) operator have been widely applied in diverse kinds of FSs, such as IFS [47], HFS [48], and NS [49]. Furthermore, Li [50] and Li et al. [51] propounded the generalized OWA operator under IFS context and established DM methodology based upon these operators. However, these operators suppose that the attributes are independent, which can lead to information loss and produce inconsistent phenomena in actual problems. Hence, scholars have concerned several special aggregation functions which can take into account the inter-relationship among diverse input arguments. The BM operator is a common operator to fuse vague information between any two variables. Wei et al. [52] presented the generalized interval neutrosophic number BM operator to construct DM approach for evaluating technological innovation capability of companies. Liu and Zhang [53] proposed several intuitionistic uncertain linguistic BM operators and utilized them to solve group decision problems. As the complexity of the assessment information and DM setting increases, the inter-relationship of all input arguments should be taken into consideration in actual applications. In light of this situation, Hara et al. [54] initially developed the Hamy mean operator, which can capture the correlation of multiarguments and have an alterable parameter that enables the decision procedure more flexible. Recently, the HM operator has been investigated to fuse fuzzy information under various fuzzy settings, such as Interval type- 2 context [55], 2-tuple linguistic neutrosophic [56], linguistic intuitionistic fuzzy number [57], and q-rung orthopair fuzzy environment [58]. Up to now, to the best of our knowledge, no study has been investigated on the HM operator under HFLS setting.

Based on the aforementioned analysis and literature review, the motivations of this article are displayed as follows: (1) the HFLS is much better than HFS and LTS to express fuzzy and indeterminate information by synthesizing the advantages of HFS and LTS; (2) the HM operator has a strength to can take into account the interrelationships of fused arguments, and it also can enable the DM model more reasonable for addressing MADM issues; and (3) the adjustable parameter of the HM operator can make the DM procedure more flexible, and the evaluator can adjust preference attitude according to it.

In view of the aforementioned comprehensive analysis, the main research objective of this article is to extend the HM operator to HFLS context and to propound several novel operators to solve MADM issues with HFL information. The contributions and goals of this study are as follows: 
(1) To extend the HM operator to HFL setting

(2) To propound the HFLHM operator, WHFLHM operator, HFLDHM operator, and WHFLDHM operator

(3) To explore the desirable properties and several particular cases of the presented operators

(4) To propose a novel MADM approach on the basis of the proposed operators

(5) To elaborate the validity and merits of the proposed approach by numerical example and comparison analysis

For the sake of the above goals, the organizational structure of this study is displayed as follows. In Section 2, we retrospect several fundamental notions including the HFS, LTS, HFLS, and HM operator. Section 3 puts forward the HFLHM and WHFLHM operator and investigates the desirable properties of them in detail. Section 4 puts forward the HFLDHM operator and WHFLDHM operator and discusses related theorems. In Section 5, a novel MADM approach is developed to tackle MADM problems based on the WHFLHM operator and WHFLDHM operator. In Section 6, an illustration example is provided to verify the validity of the proposed approach in this article, and a comparative analysis is given to manifest the advantages. The conclusion remarks are drawn in the end.

\section{Preliminaries}

In this part, we will retrospect several related fundamental definitions including HFS, LTS, HFLS, HM operator, and DHM operator.

\subsection{HFS}

Definition 1 (see [24]). Supposing that $\mathscr{X}$ is a fixed set, the mathematical expression of a HFS on $\mathscr{X}$ is depicted as follows:

$$
\mathfrak{U}=\left\{\left\langle x, h_{\mathfrak{U}}(x)\right\rangle \mid x \in \mathscr{X}\right\},
$$

in which $h_{\mathfrak{U}}(x)$ is a collection of a slice of values in $[0,1]$ which indicate the possible membership degrees. For any $x$, $h=h_{\mathfrak{U}}(x)$ is called the hesitant fuzzy element.

Definition 2 (see [35]). Assuming that $\varsigma$ be an HFE, then $\ell(\varsigma)=(1 / \#(\varsigma)) \sum_{\gamma \in \varsigma} \gamma$ is called the score function of $\varsigma$, where $\#(\varsigma)$ denotes the number of all elements in $\varsigma$. For any two HFEs $\varsigma_{1}$ and $\varsigma_{2}$, if $\ell\left(\varsigma_{1}\right)>\ell\left(\varsigma_{2}\right)$, then $\varsigma_{1}>\varsigma_{2}$; if $\ell\left(\varsigma_{1}\right)<\ell\left(\varsigma_{2}\right)$, then $\varsigma_{1}<\varsigma_{2}$.

\section{2. $\operatorname{LTS}$}

Definition 3 (see [59]). Let $\mathcal{S}=\left\{s_{\alpha} \mid \alpha=0,1, \ldots, t\right\}$ be a LTS with odd cardinality, and $s_{\alpha}$ is indicative of a possible value for a linguistic variable and meets the following characteristics:

$$
\text { (1) If } \alpha>\beta \text {, then } s_{\alpha}>s_{\beta}
$$

(2) Existing negative operators: $\operatorname{Neg}\left(s_{\alpha}\right)=s_{\beta}$, where $\alpha+\beta=t$

(3) Maximum operator: if $s_{\alpha} \geq s_{\beta}$, then $\operatorname{Max}\left\{s_{\alpha}, s_{\beta}\right\}=s_{\alpha}$

(4) Minimum operator: if $s_{\alpha} \leq s_{\beta}$, then $\operatorname{Min}\left\{s_{\alpha}, s_{\beta}\right\}=s_{\alpha}$

2.3. HFLS. Lin et al. [39] introduced the notation of HFLSs by combining the HFSs and LTSs to express fuzzy and indeterminate information more effectively.

Definition 4. Assume that $\mathscr{X}=\left\{x_{1}, x_{2}, \ldots, x_{n}\right\}$ be a referenced set. A HFLS in the mathematical form is described as

$$
\mathscr{A}=\left\{\left\langle x,\left(s_{\theta(x)}, h_{\mathscr{A}}(x)\right)\right| x \in \mathscr{X}\right\},
$$

where $h_{\mathscr{A}}(x)$ is a collection of several possible values in $[0,1]$ which indicate the possible membership degree that $x \in \mathscr{X}$ belongs to the linguistic term set $s_{\theta(x)}$, and $\theta(x) \in[0, t]$. For simplicity, $\tilde{a}=\left\langle s_{\theta(x)}, h_{\mathscr{A}}(x)\right\rangle$ is represented as a HFLE.

Example 1. Assume that $\mathscr{X}=\left\{x_{1}, x_{2}, x_{3}\right\}$ be a regular set. A HFLS on $\mathscr{X}$ is expressed as $\mathscr{A}=\left\{\left\langle x_{1}, s_{4},\{0.3\}\right\rangle,\left\langle x_{2}\right.\right.$, $\left.\left.s_{5},\{0.5,0.8\}\right\rangle,\left\langle x_{1}, s_{7},\{0.2,0.6,0.9\}\right\rangle\right\}$. HFLS $\mathscr{A}$ can be divided into three subsets which only have one goal, i.e., $\mathscr{A}$ has three HFLEs, $\left\langle s_{4},\{0.3\}\right\rangle,\left\langle s_{5},\{0.5,0.8\}\right\rangle$, and $\left\langle s_{7},\{0.2\right.$, $0.6,0.9\}\rangle$. Take $\left\langle s_{7},\{0.2,0.6,0.9\}\right\rangle$ as an example; $s_{7}$ denotes a linguistic term which can qualitatively evaluate the goal, and $\{0.2,0.6,0.9\}$ indicates some possible membership degrees that $x_{3}$ belongs to $s_{7}$. Specially, $\left\langle s_{4},\{0.3\}\right\rangle$ denotes that the degree to which $x_{1}$ belongs to $s_{4}$ which is 0.3 ; it is a particular case of HFLE, namely, fuzzy linguistic number.

As a tool that can combine the characteristic of quantitative and qualitative to express vague and uncertain information validly. HFLS synthesizes the merits of LTS and HFS, which makes the views expressed by decision makers belong to the corresponding linguistic term. LTS is more in line with the evaluation thinking of individuals, and HFE can allow multiple decision makers to give their own possible value to an alternative. Considering the complexity of evaluation information and human cognitive thinking, it is formidable for DMs to express the viewpoint with a precise numerical value. However, the HFLE can express evaluation information by a linguistic term. For instance, a linguistic term $\left(s_{5}\right)$ "good" can be utilized to evaluate performance or quality of a car. Four decision makers give their evaluation value based on the linguistic term $\left(s_{5}\right)$ "good" $\{0.2,0.3,0.4,0.6\}$, and then the evaluation information by the form of HFLE can be indicated as $\left\langle s_{5},\{0.2,0.3,0.4,0.6\}\right\rangle$. Accordingly, HFLS is a powerful and valid tool to cope with decisionmaking problems.

Definition 5 (see [39]). Let $\tilde{a}=\left\langle s_{\theta(\tilde{a})}, h(\tilde{a})\right\rangle, \tilde{a}_{1}=\left\langle s_{\theta\left(\tilde{a}_{1}\right)}\right.$, $\left.h_{\mathscr{A}}\left(\tilde{a}_{1}\right)\right\rangle$, and $\tilde{a}_{2}=\left\langle s_{\theta\left(\tilde{a}_{2}\right)}, h_{\mathscr{A}}\left(\tilde{a}_{2}\right)\right\rangle$ be three HFLEs, and $\kappa$ be a real number, then 


$$
\begin{aligned}
& \tilde{a}_{1} \bigoplus \tilde{a}_{2}=\left\langle s_{\theta\left(\tilde{a}_{1}\right)}+s_{\theta\left(\tilde{a}_{2}\right)}, \bigcup_{\gamma\left(\tilde{a}_{1}\right) \in h\left(\tilde{a}_{1}\right), \gamma\left(\tilde{a}_{2}\right) \in h\left(\tilde{a}_{2}\right)}\left\{\gamma\left(\tilde{a}_{1}\right)\right.\right. \\
&\left.\left.+\gamma\left(\tilde{a}_{2}\right)-\gamma\left(\tilde{a}_{1}\right) \gamma\left(\tilde{a}_{2}\right)\right\}\right\rangle ; \\
& \tilde{a}_{1} \otimes \tilde{a}_{2}=\left\langle s_{\theta\left(\tilde{a}_{1}\right)} \times s_{\theta\left(\tilde{a}_{2}\right)}, \bigcup_{\gamma\left(\tilde{a}_{1}\right) \in h\left(a_{1}\right), \gamma\left(\tilde{a}_{2}\right) \in h\left(\tilde{a}_{2}\right)}\left\{\gamma\left(\tilde{a}_{1}\right) \gamma\left(\tilde{a}_{2}\right)\right\}\right\rangle ; \\
& \kappa \tilde{a}=\left\langle s_{\kappa s_{\theta(\tilde{a})}}, \bigcup_{\gamma(\tilde{a}) \in h(\tilde{a})}\left\{1-(1-\gamma(\tilde{a}))^{\kappa}\right\}\right\rangle ; \\
& \tilde{a}^{\kappa}=\left\langle s_{\theta(\tilde{a})^{\kappa}}, \bigcup_{\gamma(\tilde{a}) \in h(\tilde{a})}\left\{\gamma(\tilde{a})^{\kappa}\right\}\right\rangle .
\end{aligned}
$$

In order to compare HFLEs, Lin et al. [39] gave the ranking method as follows.

Definition 6 (see [39]). Supposing that $\tilde{a}=\left\langle s_{\theta(x)}, h_{\mathscr{A}}(x)\right\rangle$ be a HFLE, and $J(\tilde{a})=\left((1 / \sharp h) \sum_{\gamma \in h} \gamma\right) s_{\theta(x)}$ is called the score function of $\tilde{a}$, where $\sharp h$ is denoted by the number of all elements in $h$. For any two HFLEs, $\tilde{a}_{1}=\left\langle s_{\theta\left(\tilde{a}_{1}\right)}, h_{\mathscr{A}}\left(\tilde{a}_{1}\right)\right\rangle$ and $\tilde{a}_{2}=\left\langle s_{\theta\left(\tilde{a}_{2}\right)}, h_{\mathscr{A}}\left(\tilde{a}_{2}\right)\right\rangle$; if $\left.J\left(\tilde{a}_{1}\right)\right\rangle J\left(\tilde{a}_{2}\right)$, then $\tilde{a}_{1}>\tilde{a}_{2}$, and if $J\left(\tilde{a}_{1}\right)=J\left(\tilde{a}_{2}\right)$, then $\tilde{a}_{1}=\tilde{a}_{2}$.

\subsection{Hamy Mean Operator}

Definition 7 (see [54]). Let $A=\left\{\widetilde{a}_{i} \mid i=1,2, \ldots, m\right\}$ be a group of positive real numbers. The definition of the $\mathrm{HM}^{(\xi)}$ operator is depicted as

$$
\operatorname{HM}^{(\xi)}\left(\tilde{a}_{1}, \tilde{a}_{2}, \ldots, \tilde{a}_{m}\right)=\frac{\sum_{1 \leq i_{1}<\cdots<i_{\xi} \leq m}\left(\prod_{j=1}^{\xi} \tilde{a}_{i_{j}}\right)^{1 / \xi}}{C_{m}^{\xi}},
$$

in which $\xi$ is a parameter, $\xi=1,2, \ldots, m, i_{1}, i_{2}, \ldots, i_{\xi}$ are $\xi$ integer values taken from the collection $\{1,2, \ldots, m\}$ of $m$ integer values, $C_{m}^{\xi}$ is the binomial coefficient, and $C_{m}^{\xi}=(m ! / \xi !(m-\xi) !)$.

The HM operator has the following properties:

(1) $\operatorname{HM}^{(\xi)}(0,0, \ldots, 0)=0$, if $\tilde{a}_{i}=0(i=1,2, \ldots, m)$

(2) $\operatorname{HM}^{(\xi)}(\widetilde{a}, \widetilde{a}, \ldots, \widetilde{a})=a$, if $\widetilde{a}_{i}=\widetilde{a}(i=1,2, \ldots, m)$

(3) $\operatorname{HM}^{(\xi)}\left(\widetilde{a}_{1}, \widetilde{a}_{2}, \ldots, \widetilde{a}_{m}\right) \leq \operatorname{HM}^{(\xi)}\left(\widetilde{b}_{1}, \widetilde{b}_{2}, \ldots, \widetilde{b}_{m}\right)$, if $\widetilde{a}_{m} \leq \widetilde{b}_{m}(i=1,2, \ldots, m)$;

(4) $\min \left\{\widetilde{a}_{i}\right\} \leq \operatorname{HM}^{(\xi)}\left(\widetilde{a}_{1}, \widetilde{a}_{2}, \ldots, \widetilde{a}_{m}\right) \leq \max \left\{\widetilde{a}_{i}\right\}$

There are two particular cases of the HM operator; numbered lists can be added as follows:
(1) When $\xi=1, \operatorname{HM}^{(\xi)}\left(\tilde{a}_{1}, \tilde{a}_{2}, \ldots, \tilde{a}_{m}\right)=(1 / m) \sum_{i=1}^{m} \tilde{a}_{i}$, and it degenerates to the arithmetic mean operator [60]

(2) When $\xi=m, \operatorname{HM}^{(\xi)}\left(\widetilde{a}_{1}, \widetilde{a}_{2}, \ldots, \widetilde{a}_{m}\right)=\left(\sum_{i=1}^{m} \widetilde{a}_{i}\right)^{1 / m}$, and it degenerates to the geometric mean operator [61]

Definition 8 (see [56]). Let $A=\left\{\widetilde{a}_{i} \mid i=1,2, \ldots, m\right\}$ be a set of positive real numbers. $\operatorname{DHM}^{(x)}$ is called the dual Hamy mean operator, which is depicted as follows:

$$
\operatorname{DHM}^{(\xi)}\left(\tilde{a}_{1}, \tilde{a}_{2}, \ldots, \tilde{a}_{m}\right)=\left(\prod_{1 \leq i_{1}<\ldots<i_{\xi} \leq m}\left(\frac{\sum_{j=1}^{\xi} a_{i_{j}}}{\xi}\right)\right)^{1 / C_{m}^{\xi}},
$$

in which $\xi$ is a parameter, $\xi=1,2, \ldots, m, i_{1}, i_{2}, \ldots, i_{\xi}$ are $\xi$ integer values taken from the set $\{1,2, \ldots, m\}$ of $m$ integer values, $C_{m}^{\xi}$ is the binomial coefficient, and $C_{m}^{\xi}=(m ! /$ $\xi !(m-\xi) !)$.

\section{Hesitant Fuzzy Linguistic Hamy Mean Aggregation Operators}

In this part, we will extend the HM operator and the DHM operator to HFL setting to present the HFLHM operator and the HFLDHM operator for aggregating HFLEs, and we further discuss particular cases and relevant properties of the proposed operators.

\subsection{HFLHM Operator}

Definition 9. Let $\mathscr{A}=\left\{\widetilde{a}_{i}=\left\langle s_{\theta\left(\tilde{a}_{i}\right)}, h_{\mathscr{A}}\left(\widetilde{a}_{i}\right)\right\rangle \mid i=1,2, \ldots, m\right\}$ be a collection of HFLEs, then the HFLHM operator is shown as

$$
\operatorname{HFLHM}^{(\xi)}\left(\widetilde{a}_{1}, \widetilde{a}_{2}, \ldots, \widetilde{a}_{m}\right)=\frac{1}{C_{m}^{\xi}}\left(\underset{1 \leq i_{1}<\cdots<i_{\xi} \leq m}{\bigoplus}\left(\bigotimes_{j=1}^{\xi} \tilde{a}_{i_{j}}\right)^{1 / \xi}\right),
$$

where $\xi$ is a parameter, $\xi=1,2, \ldots, m, i_{1}, i_{2}, \ldots, i_{\xi}$ are $\xi$ integer values taken from the set $\{1,2, \ldots, m\}$ of $m$ integer values, $C_{m}^{\xi}$ is the binomial coefficient, and $C_{m}^{\xi}=(m ! /$ $\xi !(m-\xi) !)$.

According to the operational laws of HFLE, we can obtain the following theorems of the HFLHM operator.

Theorem 1. Let $\mathscr{A}=\left\{\widetilde{a}_{i}=\left\langle s_{\theta\left(\tilde{a}_{i}\right)}, h_{\mathscr{A}}\left(\widetilde{a}_{i}\right)\right\rangle \mid i=1,2, \ldots, m\right\}$ be a collection of HFLEs; then, the fused value obtained by the HFLHM operator is also a HFLE and it can be expressed as 
Mathematical Problems in Engineering

5

$$
\begin{aligned}
\operatorname{HFLHM}^{(\xi)}\left(\widetilde{a}_{1}, \widetilde{a}_{2}, \ldots, \widetilde{a}_{m}\right)= & \frac{1}{C_{m}^{\xi}}\left(\bigoplus_{1 \leq i_{1}<\ldots<i_{\xi} \leq m}\left(\bigotimes_{j=1}^{\xi} \widetilde{a}_{i_{j}}\right)^{1 / \xi}\right), \\
& \cdot\left\langle s_{\left(1 / C_{m}^{\xi}\right)\left(\sum_{1 \leq i_{1}<\cdots<i_{\xi} \leq m}\left(\prod_{j=1}^{\xi} \theta\left(\widetilde{a}_{i_{j}}\right)\right)^{1 / \xi}\right),}\right. \\
& \left.\cdot\left(\bigcup_{\gamma\left(\widetilde{a}_{i}\right) \in h\left(\widetilde{a}_{i}\right)(i=1,2, \ldots, m)}\left\{1-\left(\prod_{1 \leq i_{1}<\ldots<i_{\xi} \leq m}\left(1-\left(\prod_{j=1}^{\xi} \gamma\left(\widetilde{a}_{i_{j}}\right)\right)^{1 / \xi}\right)\right)^{1 / C_{m}^{\xi}}\right\}\right)\right\rangle .
\end{aligned}
$$

Proof Therefore,

(1) Firstly, we prove equation (7) holds, and according to the operational laws of HFLEs, we have

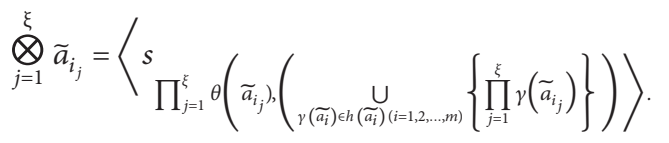

$$
\left(\bigotimes_{j=1}^{\xi} \tilde{a}_{i_{j}}\right)^{1 / \xi}=\left\langle s\left(\prod_{j=1}^{\xi} \theta\left(\tilde{a}_{i_{j}}\right)\right)^{1 / \xi},\left(\bigcup_{\gamma\left(\tilde{a}_{i}\right) \in h\left(\tilde{a}_{i}\right)(i=1,2, \ldots, m)}\left\{\left(\prod_{j=1}^{\xi} \gamma\left(\tilde{a}_{i_{j}}\right)\right)^{1 / \xi}\right\}\right)\right\rangle .
$$

Furthermore,

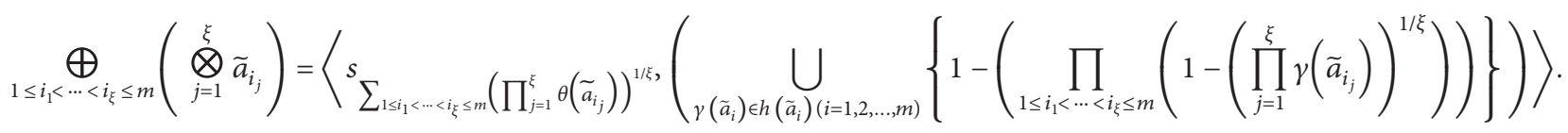

(10)

Then,

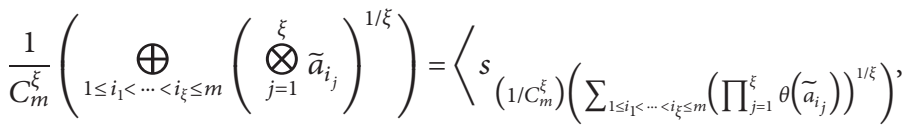

$$
\begin{aligned}
& \left.\cdot\left(\bigcup_{\gamma\left(\widetilde{a}_{i}\right) \in h\left(\widetilde{a}_{i}\right)(i=1,2, \ldots, m)}\left\{1-\left(\prod_{1 \leq i_{1}<\ldots<i_{\xi} \leq m}\left(1-\left(\prod_{j=1}^{\xi} \gamma\left(\tilde{a}_{i_{j}}\right)\right)^{1 / \xi}\right)\right)^{1 / C_{m}^{\xi}}\right\}\right)\right\rangle .
\end{aligned}
$$


Hence,

$$
\begin{aligned}
& \operatorname{HFLHM}^{\xi}\left(\widetilde{a}_{1}, \widetilde{a}_{2}, \ldots, \widetilde{a}_{m}\right)=\left\langle s_{\left(1 / C_{m}^{\xi}\right.}\right)\left(\sum_{1 \leq i_{1}<\ldots<i_{\xi} \leq m}\left(\prod_{j=1}^{\xi} \theta\left(\tilde{a}_{i_{j}}\right)\right)^{1 / \xi}\right), \\
& \left.\cdot\left(\bigcup_{\gamma\left(\tilde{a}_{i}\right) \in h\left(\tilde{a}_{i}\right)(i=1,2, \ldots, m)}\left\{1-\left(\prod_{1 \leq i_{1}<\ldots<i_{\xi} \leq m}\left(1-\left(\prod_{j=1}^{\xi} \gamma\left(\tilde{a}_{i_{j}}\right)\right)^{1 / \xi}\right)\right)^{1 / C_{m}^{\xi}}\right\}\right)\right\rangle .
\end{aligned}
$$

(2) Secondly, we prove equation (7) is a HFLE. It is known that $\tilde{a}_{i_{j}} \in[0,1]$, and we can acquire

$$
\begin{aligned}
\prod_{j=1}^{\xi} \gamma\left(\tilde{a}_{i_{j}}\right) \in[0,1] & \Longrightarrow\left(\prod_{j=1}^{\xi} \gamma\left(\tilde{a}_{i_{j}}\right)\right)^{1 / \xi} \in[0,1] \Longrightarrow 1-\left(\prod_{j=1}^{\xi} \gamma\left(\tilde{a}_{i_{j}}\right)\right)^{1 / \xi} \in[0,1] \\
& \Longrightarrow \prod_{1 \leq i_{1}<\ldots<i_{\xi} \leq m}\left(1-\left(\prod_{j=1}^{\xi} \gamma\left(\tilde{a}_{i_{j}}\right)\right)^{1 / \xi}\right) \in[0,1] \Longrightarrow\left(\prod_{1 \leq i_{1}<\ldots<i_{\xi} \leq m}\left(1-\left(\prod_{j=1}^{\xi} \gamma\left(\tilde{a}_{i_{j}}\right)\right)^{1 / \xi}\right)\right)^{1 / C_{m}^{\xi}} \\
& \Longrightarrow 1-\left(\prod_{1 \leq i_{1}<\ldots<i_{\xi} \leq m}\left(1-\left(\prod_{j=1}^{\xi} \gamma\left(\tilde{a}_{i_{j}}\right)\right)^{1 / \xi}\right)\right)^{1 / C_{m}^{\xi}} \in[0,1] .
\end{aligned}
$$

Example 2. Let $\widehat{a}_{1}=\left\langle s_{3},\{0.2,0.3\}\right\rangle, \widehat{a}_{2}=\left\langle s_{4},\{0.4,0.5\}\right\rangle$, and $\hat{a}_{3}=\left\langle s_{6},\{0.6,0.7\}\right\rangle$ be three HFLEs, then the aggregation value of them can be obtained by the HFLHM operator, which is shown as follows (suppose $\xi=2$ ):

$$
\begin{aligned}
& \operatorname{HFLHM}^{(\xi)}\left(\tilde{a}_{1}, \tilde{a}_{2}, \tilde{a}_{3}\right) \\
& =\left\langle s_{\left(1 / C_{k}^{\xi}\right)}\left(\sum_{1 \leq i_{1}<\ldots<i_{\xi} \leq m}\left(\prod_{j=1}^{\xi} \theta\left(\tilde{a}_{i_{j}}\right)\right)^{1 / \xi}\right)\right. \\
& \gamma\left(\tilde{a}_{i}\right) \in h\left(\tilde{a}_{i}\right)(i=1,2, \ldots, m) \\
& =\left\langle s_{4.2019},\{0.3793,0.4044,0.4131,0.4409,0.4232,0.4496,0.4576,0.4863\}\right\rangle .
\end{aligned}
$$

Hereinbelow, we will talk about several desired theorems

$$
\operatorname{HFLHM}^{(\xi)}\left(\widetilde{a}_{1}, \widetilde{a}_{2}, \ldots, \widetilde{a}_{m}\right)=\widetilde{a} .
$$

\section{of the HFLHM operator.}

Theorem 2 (idempotency). Let $\mathscr{A}=\left\{\tilde{a}_{i}=\left\langle s_{\theta\left(\tilde{a}_{i}\right)}, h_{\mathscr{A}}\left(\tilde{a}_{i}\right)\right\rangle\right.$ $\mid i=1,2, \ldots, m\}$ be a collection of HFLEs; if $\tilde{a}_{i}=\widetilde{a}=$ $\left\langle s_{\theta(\tilde{a})}, h_{\mathscr{A}}(\tilde{a})\right\rangle(i=1,2, \ldots, m)$, then
Proof. Since $\tilde{a}=\left\langle s_{\theta(\tilde{a})}, h_{\mathscr{A}}(\tilde{a})\right\rangle$, on the basis of Theorem 1 , we have

$$
\begin{aligned}
& \operatorname{HFLHM}^{(\xi)}\left(\tilde{a}_{1}, \tilde{a}_{2}, \ldots, \tilde{a}_{m}\right) \\
& =\left\langle s_{\left(1 / C_{m}^{\xi}\right)}\left(\sum_{1 \leq i_{1}<\ldots<i \xi \leq m}\left(\prod_{j=1}^{\xi} \theta\left(\tilde{a}_{i_{j}}\right)\right)^{1 / \xi}\right),\left(\bigcup_{\gamma(\tilde{a}) \in h(\tilde{a})}\left\{1-\left(\prod_{1 \leq i_{1}<\ldots<i_{\xi} \leq m}\left(1-\left(\prod_{j=1}^{\xi} \gamma\left(\tilde{a}_{i_{j}}\right)\right)^{1 / \xi}\right)\right)^{1 / C_{m}^{\xi}}\right\}\right)\right\rangle
\end{aligned}
$$




$$
\begin{aligned}
& =\left\langle s_{\left(1 / C_{m}^{\xi}\right)}\left(\sum_{1 \leq i_{1}<\ldots<i_{\xi} \leq m}\left(\theta\left(\tilde{a}_{i_{j}}\right)\right)^{1 / \xi}\right),\left(\bigcup_{\gamma(\widetilde{a}) \in h(\widetilde{a})}\left\{1-\left(\prod_{1 \leq i_{1}<\cdots<i_{\xi} \leq m}\left(1-\left(1-\left((\gamma(\widetilde{a}))^{\xi}\right)\right)^{1 / \xi}\right)\right)^{1 / C_{m}^{\xi}}\right\}\right)\right\rangle \\
& =\left\langle s_{\left(1 / C_{m}^{\xi}\right)}\left(C_{m}^{\xi} \theta(\widetilde{a})\right),\left(\bigcup_{\gamma(\tilde{a}) \in h(\tilde{a})}\left\{1-\left((1-\gamma(\widetilde{a}))^{C_{m}^{\xi}}\right)^{1 / C_{m}^{\xi}}\right\}\right)\right\rangle \\
& =\left\langle s_{\theta(\tilde{a})},\left(\bigcup_{\gamma(\tilde{a}) \in h(\tilde{a})}\{1-(1-\gamma(\widetilde{a}))\}\right)\right\rangle=\left\langle s_{\theta(\tilde{a})},\left(\bigcup_{\gamma(\tilde{a}) \in h(\tilde{a})}\{\gamma(\widetilde{a})\}\right)\right\rangle=\left\langle s_{\theta(\tilde{a})}, h(\tilde{a})\right\rangle=\tilde{a} .
\end{aligned}
$$

Theorem 3 (monotonicity). Let $\mathscr{A}=\left\{\widetilde{a}_{i}=\left\langle s_{\theta\left(\tilde{a}_{i}\right)}, h_{\mathscr{A}}\left(\tilde{a}_{i}\right)\right\rangle\right.$ $\mid i=1,2, \ldots, m\}$ and $\mathscr{B}=\left\{\tilde{b}_{i}=\left\langle s_{\theta\left(\tilde{b}_{i}\right)}, h_{\mathscr{B}}\left(\tilde{b}_{i}\right)\right\rangle \mid i=1,2\right.$, $\ldots, m\}$ be two set of HFLEs; if $\theta\left(\tilde{a}_{i}\right) \geq \theta\left(\tilde{b}_{i}\right)$ and $h_{\mathscr{A}}\left(\widetilde{a}_{i}\right) \geq h_{\mathscr{B}}\left(\widetilde{b}_{i}\right)$ for each $i(i=1,2, \ldots, m)$, then

$$
\operatorname{HFLHM}^{(\xi)}\left(\widetilde{a}_{1}, \tilde{a}_{2}, \ldots, \tilde{a}_{m}\right) \geq \operatorname{HFLHM}^{(\xi)}\left(\widetilde{b}_{1}, \tilde{b}_{2}, \ldots, \tilde{b}_{m}\right) \text {. }
$$

Proof. Since $\theta\left(\widetilde{a}_{i}\right) \geq \theta\left(\widetilde{b}_{i}\right)$, we have

$$
\prod_{j=1}^{\xi} \theta\left(\tilde{a}_{i_{j}}\right) \geq \prod_{j=1}^{\xi} \theta\left(\tilde{b}_{i_{j}}\right),\left(\prod_{j=1}^{\xi} \theta\left(\tilde{a}_{i_{j}}\right)\right)^{1 / \xi} \geq\left(\prod_{j=1}^{\xi} \theta\left(\tilde{b}_{i_{j}}\right)\right)^{1 / \xi} .
$$

Then,

$$
\left(\sum_{1 \leq i_{1}<\cdots<i_{\xi} \leq m}\left(\prod_{j=1}^{\xi} \theta\left(\tilde{a}_{i_{j}}\right)\right)^{1 / \xi}\right) \geq\left(\sum_{1 \leq i_{1}<\cdots<i_{\xi} \leq m}\left(\prod_{j=1}^{\xi} \theta\left(\tilde{b}_{i_{j}}\right)\right)^{1 / \xi}\right) \text {. }
$$

Accordingly,

$$
\begin{aligned}
& \frac{1}{C_{m}^{\xi}}\left(\sum_{1 \leq i_{1}<\cdots<i_{\xi} \leq m}\left(\prod_{j=1}^{\xi} \theta\left(\tilde{a}_{i_{j}}\right)\right)^{1 / \xi}\right) \\
& \quad \geq \frac{1}{C_{m}^{\xi}}\left(\sum_{1 \leq i_{1}<\cdots<i_{\xi} \leq m}\left(\prod_{j=1}^{\xi} \theta\left(\tilde{b}_{i_{j}}\right)\right)^{1 / \xi}\right),
\end{aligned}
$$

that is, $s_{\theta\left(\tilde{a}_{i}\right)} \geq s_{\theta\left(\tilde{b}_{i}\right)}$.

Since $h\left(\tilde{a}_{i}\right) \geq h\left(\widetilde{b}_{i}\right)$ and $\gamma\left(\widetilde{a}_{i_{j}}\right) \geq \gamma\left(\widetilde{b}_{i_{j}}\right)$, we have

$$
\prod_{j=1}^{\xi} \gamma\left(\tilde{a}_{i_{j}}\right) \geq \prod_{j=1}^{\xi} \gamma\left(\tilde{b}_{i_{j}}\right)
$$

$$
1-\left(\prod_{j=1}^{\xi} \gamma\left(\widetilde{a}_{i_{j}}\right)\right)^{1 / \xi} \leq 1-\left(\prod_{j=1}^{\xi} \gamma\left(\widetilde{b}_{i_{j}}\right)\right)^{1 / \xi}
$$

Then,

$$
\prod_{1 \leq i_{1}<\cdots<i_{\xi} \leq m}\left(1-\left(\prod_{j=1}^{\xi} \gamma\left(\tilde{a}_{i_{j}}\right)\right)^{1 / \xi}\right) \leq \prod_{1 \leq i_{1}<\cdots<i_{\xi} \leq m}\left(1-\left(\prod_{j=1}^{\xi} \gamma\left(\tilde{b}_{i_{j}}\right)\right)^{1 / \xi}\right) .
$$

Therefore,

$$
1-\left(\prod_{1 \leq i_{1}<\ldots<i_{\xi} \leq m}\left(1-\left(\prod_{j=1}^{\xi} \gamma\left(\tilde{a}_{i_{j}}\right)\right)^{1 / \xi}\right)\right)^{1 / C_{m}^{\xi}} \geq 1-\left(\prod_{1 \leq i_{1}<\ldots<i_{\xi} \leq m}\left(1-\left(\prod_{j=1}^{\xi} \gamma\left(\tilde{b}_{i_{j}}\right)\right)^{1 / \xi}\right)\right)^{1 / C_{m}^{\xi}}
$$

Besides, let $J(\widetilde{a})=\left((1 / \sharp h) \sum_{\gamma \in h} \gamma\right) J_{\theta(\tilde{a})}$ and $J(\widetilde{b})=\left((1 / \sharp h) \sum_{\gamma \in h_{5}} \gamma\right) J_{\theta(\bar{b})}$ be the score function of $\widetilde{a}$ and $\widetilde{b}$, respectively. Based on the above analysis, we have $J(\widetilde{a})$ $\geq J(\tilde{b})$, that is, $\operatorname{HFLHM}^{(\xi)}\left(\tilde{a}_{1}, \tilde{a}_{2}, \ldots, \tilde{a}_{m}\right) \geq \operatorname{HFLHM}^{(\xi)}\left(\tilde{b}_{1}\right.$, $\left.\widetilde{b}_{2}, \ldots, \widetilde{b}_{m}\right)$.
Theorem 4 (boundedness). Assuming that $\mathscr{A}=\left\{\widetilde{a}_{i}=\left\langle s_{\theta\left(\tilde{a}_{i}\right)}, h_{\mathscr{A}}\left(\widetilde{a}_{i}\right)\right\rangle \mid i=1,2, \ldots, m\right\}$ be a collection of HFLE. Assume that $\tilde{a}^{-}=\min \left(\tilde{a}_{i}, \tilde{a}_{2}, \ldots, \tilde{a}_{m}\right)$ and $\tilde{a}^{+}=\max \left(\tilde{a}_{i}, \widetilde{a}_{2}, \ldots, \tilde{a}_{m}\right)$, then

$$
\widetilde{a}^{-} \leq \operatorname{HFLHM}^{(\xi)}\left(\widetilde{a}_{1}, \widetilde{a}_{2}, \ldots, \widetilde{a}_{m}\right) \leq \tilde{a}^{+} .
$$


Proof. According to Theorem 3, we have

$$
\begin{aligned}
& \operatorname{HFLHM}^{(\xi)}\left(\tilde{a}^{-}, \tilde{a}^{-}, \ldots, \tilde{a}^{-}\right) \leq \operatorname{HFLHM}^{(\xi)}\left(\tilde{a}_{1}, \tilde{a}_{2}, \ldots, \tilde{a}_{m}\right) \\
& \quad \leq \operatorname{HFLHM}^{(\xi)}\left(\tilde{a}^{+}, \tilde{a}^{+}, \ldots, \tilde{a}^{+}\right) .
\end{aligned}
$$

According to Theorem 2, we have

$$
\begin{gathered}
\operatorname{HFLHM}^{(\xi)}\left(\widetilde{a}^{-}, \tilde{a}^{-}, \ldots, \widetilde{a}^{-}\right)=\widetilde{a}^{-}, \\
\operatorname{HFLHM}^{(\xi)}\left(\widetilde{a}^{+}, \tilde{a}^{+}, \ldots, \tilde{a}^{+}\right)=\widetilde{a}^{+} .
\end{gathered}
$$

Hence, we obtain

$$
\tilde{a}^{-} \leq \operatorname{HFLHM}^{(\xi)}\left(\tilde{a}_{1}, \tilde{a}_{2}, \ldots, \tilde{a}_{m}\right) \leq \tilde{a}^{+} .
$$

Theorem 5 (commutativity). Assume $\tilde{a}_{i}=\left\langle s_{\theta\left(\tilde{a}_{i}\right)}, h\right.$ $\left.\left(\tilde{a}_{i}\right)\right\rangle(i=1,2, \ldots, m)$ and $\tilde{b}_{i}=\left\langle s_{\theta\left(\tilde{b}_{i}\right)}, h\left(\tilde{b}_{i}\right)\right\rangle(i=1,2, \ldots$, $m)$ be a collection of HFLEs. Assume that $\left(\widetilde{b}_{1}, \widetilde{b}_{2}, \ldots, \widetilde{b}_{m}\right)$ is any permutation of $\left(\widetilde{a}_{1}, \widetilde{a}_{2}, \ldots, \widetilde{a}_{m}\right)$, then

$$
\operatorname{HFLHM}^{(\xi)}\left(\tilde{a}_{1}, \widetilde{a}_{2}, \ldots, \tilde{a}_{m}\right)=\operatorname{HFLHM}^{(\xi)}\left(\widetilde{b}_{1}, \tilde{b}_{2}, \ldots, \tilde{b}_{m}\right) \text {. }
$$

Proof. Since $\left(\tilde{b}_{1}, \tilde{b}_{2}, \ldots, \tilde{b}_{m}\right)$ is any permutation of $\left(\widetilde{a}_{1}, \widetilde{a}_{2}, \ldots, \widetilde{a}_{m}\right)$, then

$$
\begin{aligned}
\frac{1}{C_{m}^{\xi}}\left(\underset{1 \leq i_{1}<\cdots<i_{\xi} \leq m}{\bigoplus}\left(\bigotimes_{j=1}^{\xi} \tilde{a}_{i_{j}}\right)^{1 / \xi}\right)= & \frac{1}{C_{m}^{\xi}}\left(\underset{1 \leq i_{1}<\cdots<i_{\xi} \leq m}{\bigoplus}\right. \\
& \left.\cdot\left(\bigotimes_{j=1}^{\xi} \tilde{b}_{i_{j}}\right)^{1 / \xi}\right) .
\end{aligned}
$$

Accordingly, $\operatorname{HFLHM}^{(\xi)}\left(\widetilde{a}_{1}, \tilde{a}_{2}, \ldots, \tilde{a}_{m}\right)=\operatorname{HFLHM}^{(\xi)}$ $\left(\tilde{b}_{1}, \tilde{b}_{2}, \ldots, \tilde{b}_{m}\right)$.

In what follows, we shall explore some particular cases of the HFLHM operator obtained by assigning different values of parameter $\xi$.

Case 1. When $\xi=1$, the HFLHM operator shall degenerate to the hesitant fuzzy linguistic arithmetic average operator.

$$
\operatorname{HFLHM}^{(1)}\left(\tilde{a}_{1}, \tilde{a}_{2}, \ldots, \tilde{a}_{m}\right)=\frac{1}{m} \sum_{i=1}^{m} \tilde{a}_{i}=\left\langle s_{(1 / m) \sum_{i=1}^{m} \theta \tilde{a}_{i}, \underset{\gamma\left(\tilde{a}_{i}\right) \in h\left(\tilde{a}_{i}\right)(i=1,2, \ldots, m)}{\bigcup}}^{\bigcup}\left\{1-\prod_{i=1}^{m}\left(1-\gamma\left(\tilde{a}_{i_{j}}\right)\right)^{1 / m}\right\}\right\rangle .
$$

Case 2. When $\xi=m$, the HFLHM operator shall degenerate to the hesitant fuzzy linguistic geometric average operator.

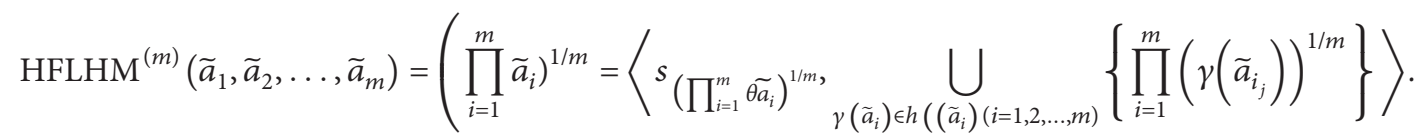

3.2. Hesitant Fuzzy Linguistic Weighted Hamy Mean Operator. The attribute weights play an essential role in actual decision-making issues, and they shall affect the ultimate decision results. Accordingly, it is indispensable to take into consideration the weights of attribute during the information fusion course. So, we will present the WHFLHM operator which is as follows.
Definition 10 . Let $\mathscr{A}=\left\{\widetilde{a}_{i}=\left\langle s_{\theta\left(\tilde{a}_{i}\right)}, h_{\mathscr{A}}\left(\widetilde{a}_{i}\right)\right\rangle \mid i=1,2\right.$, $\ldots, m\}$ be a collection of HFLEs, and $w=\left\{w_{1}, w_{2}, \ldots, w_{m}\right\}^{T}$ is the associated weight vector of $\tilde{a}_{i}(i=1,2, \ldots, m)$ such that $w_{i} \in[0,1]$ and $\sum_{i=1}^{m} w_{i}=1$. Then, the WHFLHM operator is described as follows:

$$
\mathrm{WHFLHM}_{w}^{(\xi)}=\left(\tilde{a}_{1}, \tilde{a}_{2}, \ldots, \tilde{a}_{m}\right)= \begin{cases}\frac{1}{C_{m-1}^{\xi}}\left(\underset{1 \leq i_{1}<\cdots<i_{\xi} \leq m}{\bigoplus}\left(1-\sum_{j=1}^{\xi} w_{i_{j}}\right)\left(\bigotimes_{j=1}^{\xi} \widetilde{a}_{i_{j}}\right)^{1 / \xi}\right), & 1 \leq \xi \leq m, \\ \bigotimes_{i=1}^{\xi} \tilde{a}_{i}^{\left(1-w_{i}\right) /(m-1)}, & \xi=m,\end{cases}
$$

where $\xi$ is a parameter, $\xi=1,2, \ldots, m, i_{1}, i_{2}, \ldots, i_{\xi}$ are $\xi$ integer values taken from the set $\{1,2, \ldots, m\}$ of $m$ integer values, $C_{m}^{\xi}$ is the binomial coefficient, and $C_{m}^{\xi}=(m ! /$ $\xi !(m-\xi) !)$. 
Theorem 6. Let $\mathscr{A}=\left\{\widetilde{a}_{i}=\left\langle s_{\theta\left(\tilde{a}_{i}\right)}, h_{\mathscr{A}}\left(\widetilde{a}_{i}\right)\right\rangle \mid i=1,2, \ldots, m\right\}$ be a collection of HFLEs, and the associated weight vector $w_{i} \in[0,1]$ and $\sum_{i=1}^{m} w_{i}=1$. Then, the aggregation result obtained by Definition 10 is also a HFLE, and it can be expressed as

$$
\begin{aligned}
& \operatorname{WHFLHM}_{w}^{(\xi)}=\left(\widetilde{a}_{1}, \tilde{a}_{2}, \ldots, \widetilde{a}_{m}\right)=\frac{1}{C_{m-1}^{\xi}}\left(\underset{1 \leq i_{1} \leq \ldots<i_{\xi} \leq m}{\bigoplus_{j=1}}\left(1-\sum_{j=1}^{\xi} w_{i_{j}}\right)\left(\bigotimes_{j=1}^{\xi} \tilde{a}_{i_{j}}\right)^{1 / \xi}\right) \\
& =\left\langle s_{1 / C_{m-1}^{\xi}}\left(\sum_{1 \leq i_{1} \ldots \ldots i i_{\xi} \leq m}\left(1-\sum_{j=1}^{\xi} w_{i_{j}}\right)\left(\prod_{j=1}^{\xi} \theta\left(\tilde{a}_{i_{j}}\right)\right)^{1 / \xi}\right)^{\prime}\right. \\
& \left.\cdot\left(\bigcup_{\gamma\left(\tilde{a}_{i}\right) \in h\left(\tilde{a}_{i}\right)(i=1,2, \ldots, m)}\left\{1-\left(\prod_{1 \leq i_{1}<\ldots<i_{\xi} \leq m}\left(1-\left(\prod_{j=1}^{\xi} \gamma\left(\tilde{a}_{i_{j}}\right)\right)^{1 / \xi}\right)^{\left(1-\sum_{j=1}^{\xi} w_{i_{j}}\right)}\right)^{1 / C_{m-1}^{\xi}}\right\}\right)\right\rangle \quad(1 \leq \xi<m),
\end{aligned}
$$

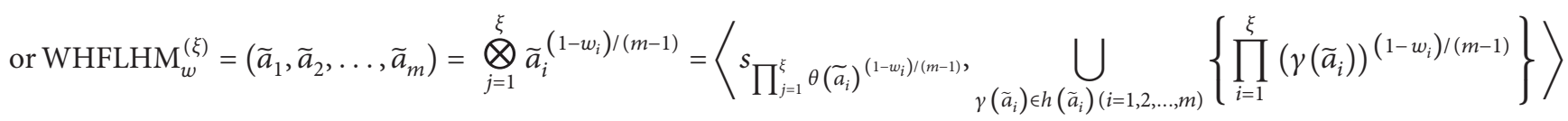

(1) Firstly, we prove equation (33) holds. For the first situation, when $1 \leq \xi \leq m$, according to the operational laws of HFLEs, we have

$$
\bigotimes_{j=1}^{\xi} \widetilde{a}_{i_{j}}=\left\langle s \prod_{j=1}^{\xi} \theta\left(\tilde{a}_{i_{j}}\right) \bigcup_{\gamma\left(\tilde{a}_{i}\right) \in h\left(\tilde{a}_{i}\right)(i=1,2, \ldots, m)}\left\{\prod_{j=1}^{\xi} \gamma\left(\tilde{a}_{i_{j}}\right)\right\}\right\rangle \text {. }
$$

$$
\begin{aligned}
\left(\bigotimes_{j=1}^{\xi} \tilde{a}_{i_{j}}\right)^{1 / \xi}= & \left\langle s\left(\prod_{j=1}^{\xi} \theta\left(\tilde{a}_{i_{j}}\right)\right)^{1 / \xi}, \bigcup_{\gamma\left(\tilde{a}_{i}\right) \in h\left(\tilde{a}_{i}\right)(i=1,2, \ldots, m)}\right. \\
& \left.\cdot\left\{\left(\prod_{j=1}^{\xi} \gamma\left(\tilde{a}_{i_{j}}\right)\right)^{1 / \xi}\right\}\right\rangle .
\end{aligned}
$$

Furthermore,

Therefore,

$$
\left(1-\sum_{j=1}^{\xi} w_{i_{j}}\right)\left(\bigotimes_{j=1}^{\xi} \tilde{a}_{i_{j}}\right)^{1 / \xi}=\left\langle s\left(1-\sum_{j=1}^{\xi} w_{i_{j}}\right)\left(\prod_{j=1}^{\xi} \theta\left(\tilde{a}_{i_{j}}\right)\right)^{1 / \xi}, \bigcup_{\gamma\left(\tilde{a}_{i}\right) \in h\left(\tilde{a}_{i}\right)(i=1,2, \ldots, m)}\left\{1-\left(1-\left(\prod_{j=1}^{\xi} \gamma\left(\tilde{a}_{i_{j}}\right)\right)^{1 / \xi}\right)^{\left(1-\sum_{j=1}^{\xi} w_{i_{j}}\right)}\right\}\right\rangle .
$$

Moreover,

$$
\begin{aligned}
& \bigoplus_{1 \leq i_{1}<\cdots<i_{\xi} \leq m}\left(1-\sum_{j=1}^{\xi} w_{i_{j}}\right)\left(\bigotimes_{j=1}^{\xi} \tilde{a}_{i_{j}}\right)^{1 / \xi} \\
& =\left\langle s \sum_{1 \leq i_{1}<\ldots<i_{\xi} \leq m}\left(1-\sum_{j=1}^{\xi} w_{i_{j}}\right)\left(\prod_{j=1}^{\xi} \theta\left(\tilde{a}_{i_{j}}\right)\right)^{1 / \xi}, \underset{\gamma\left(\tilde{a}_{i}\right) \in h\left(\tilde{a}_{i}\right)(i=1,2, \ldots, m)}{\bigcup}\left\{1-\left(\prod_{1 \leq i_{1}<\ldots<i_{\xi} \leq m}\left(1-\left(\prod_{j=1}^{\xi} \gamma\left(\tilde{a}_{i_{j}}\right)\right)^{1 / \xi}\right)^{\left(1-\sum_{j=1}^{\xi} w_{i_{j}}\right)}\right)^{1 / C_{m-1}^{\xi}}\right\}\right\rangle .
\end{aligned}
$$


Accordingly,

$$
\begin{aligned}
& \frac{1}{C_{m-1}^{\xi}}\left(\bigoplus_{1 \leq i_{1}<\ldots<i_{\xi} \leq m}\left(1-\sum_{j=1}^{\xi} w_{i_{j}}\right)\left(\bigotimes_{j=1}^{\xi} \tilde{a}_{i_{j}}\right)^{1 / \xi}\right) \\
& =\left\langle s_{\left(1 / C_{m-1}^{\xi}\right)\left(\sum_{1 \leq i_{1}<\ldots<i_{\xi} \leq m}\left(1-\sum_{j=1}^{\xi} w_{i_{j}}\right)\left(\prod_{j=1}^{\xi} \theta\left(\tilde{a}_{i_{j}}\right)\right)^{1 / \xi}\right),}\right. \\
& \quad \bigcup_{\gamma\left(\tilde{a}_{i}\right) \in h\left(\tilde{a}_{i}\right)(i=1,2, \ldots, m)}\left\{1-\left(\prod_{1 \leq i_{1}<\ldots<i_{\xi} \leq m}\left(1-\left(\prod_{j=1}^{\xi} \gamma\left(\tilde{a}_{i_{j}}\right)\right)^{1 / \xi}\right)^{\left.\left.\left.\left(1-\sum_{j=1}^{\xi} w_{i_{j}}\right)\right)^{1 / C_{m-1}^{\xi}}\right\}\right\rangle .}\right.\right.
\end{aligned}
$$

For the second situation, when $\xi=m$, we have

$$
\begin{aligned}
\tilde{a}_{i}^{\left(1-w_{i}\right) /(m-1)}=\left\langle s_{\theta\left(\tilde{a}_{i}\right)^{\left(1-w_{i}\right) /(m-1)},}\right. & \left.\left\{\bigcup_{\gamma\left(\tilde{a}_{i}\right) \in h\left(\tilde{a}_{i}\right)(i=1,2, \ldots, m)}\left\{\left(\widetilde{a}_{i}\right)\right)^{\left(1-w_{i}\right) /(m-1)}\right\}\right\rangle .
\end{aligned}
$$

$$
\begin{aligned}
\bigotimes_{i=1}^{\xi} \tilde{a}_{i}^{\left(1-w_{i}\right) /(m-1)}= & \left\langle s \prod_{i=1}^{\xi} \theta\left(\widetilde{a}_{i}\right)^{\left(1-w_{i}\right) /(m-1)}, \bigcup_{\gamma\left(\tilde{a}_{i}\right) \in h\left(\tilde{a}_{i}\right)(i=1,2, \ldots, m)}\right. \\
& \left.\cdot\left\{\prod_{i=1}^{\xi} \gamma\left(\widetilde{a}_{i}\right)^{\left(1-w_{i}\right) /(m-1)}\right\}\right\rangle .
\end{aligned}
$$

(2) Secondly, we prove equation (33) is a HFLE. It is known that $\gamma\left(\widetilde{a}_{i_{j}}\right) \in[0,1]$; when $(1 \leq \xi \leq m)$, we can acquire

Hence,

$$
\begin{gathered}
\prod_{j=1}^{\xi} \gamma\left(\tilde{a}_{i_{j}}\right) \in[0,1] \Longrightarrow\left(\prod_{j=1}^{\xi} \gamma\left(\tilde{a}_{i_{j}}\right)\right)^{1 / \xi} \in[0,1] \Longrightarrow 1-\left(\prod_{j=1}^{\xi} \gamma\left(\tilde{a}_{i_{j}}\right)\right)^{1 / \xi} \in[0,1] \\
\Longrightarrow \prod_{1 \leq i_{1}<\ldots<i_{\xi} \leq m}\left(1-\left(\prod_{j=1}^{\xi} \gamma\left(\tilde{a}_{i_{j}}\right)\right)^{1 / \xi}\right)^{\left(1-\sum_{j=1}^{\xi} w_{i_{j}}\right)} \in[0,1] \Longrightarrow \prod_{1 \leq i_{1}<\ldots<i_{\xi} \leq m}\left(\left(1-\left(\prod_{j=1}^{\xi} \gamma\left(\tilde{a}_{i_{j}}\right)\right)^{1 / \xi}\right)^{\left.\left(1-\sum_{j=1}^{\xi} w_{i_{j}}\right)\right)^{1 / C_{m-1}^{\xi}} \in[0,1]}\right. \\
\Longrightarrow\left(1-\left(\prod_{1 \leq i_{1}<\cdots<i_{\xi} \leq m}\left(\left(1-\left(\prod_{j=1}^{\xi} \gamma\left(\tilde{a}_{i_{j}}\right)\right)^{1 / \xi}\right)^{\left(1-\sum_{j=1}^{\xi} w_{i_{j}}\right)}\right)^{1 / C_{m-1}^{\xi}}\right)\right) \in[0,1] .
\end{gathered}
$$

For the other situation, when $\xi=m$, it is easy to prove, so we omit the proof process here.

In the following, we will investigate some desirable properties of the WHFLHM operator.

Theorem 7 (idempotency). Let $\mathscr{A}=\left\{\widetilde{a}_{i}=\left\langle s_{\theta\left(\tilde{a}_{i}\right)}, h_{\mathscr{A}}\left(\widetilde{a}_{i}\right)\right\rangle \mid\right.$ $i=1,2, \ldots, m\}$ be a collection of HFLEs, and $w=\left\{w_{1}, w_{2}\right.$, $\left.\ldots, w_{m}\right\}^{T}$ is the associated weight vector of $\widetilde{a}_{i}(i=1,2, \ldots, m)$ such that $w_{i} \in[0,1]$ and $\sum_{i=1}^{m} w_{i}=1$. If $\tilde{a}_{i}=\tilde{a}=$ $\left\langle s_{\theta(\tilde{a})}, h_{\mathscr{A}}(\widetilde{a})\right\rangle(i=1,2, \ldots, m)$, then

$$
\operatorname{WHFLHM}_{w}^{(\xi)}\left(\widetilde{a}_{1}, \tilde{a}_{2}, \ldots, \tilde{a}_{m}\right)=\tilde{a} .
$$

Proof. Since $\widetilde{a}=\left\langle s_{\theta(\widetilde{a})}, h_{\mathscr{A}}(\widetilde{a})\right\rangle$, based on Theorem 6, we have 
(1) For the first situation, when $1 \leq \xi \leq m$,

$$
\begin{aligned}
& \operatorname{WHFLHM}_{w}^{(\xi)}=\left(\widetilde{a}_{1}, \widetilde{a}_{2}, \ldots, \widetilde{a}_{m}\right)=\frac{1}{C_{m-1}^{\xi}}\left(\underset{1 \leq i_{1}<\cdots<i_{\xi} \leq m}{\bigoplus_{j=1}}\left(1-\sum_{i_{j}}^{\xi} w_{j}\right)\left(\bigotimes_{j=1}^{\xi} \tilde{a}_{i_{j}}\right)^{1 / \xi}\right) \\
& =\left\langle s_{\left(1 / C_{m-1}^{\xi}\right)}\left(\sum_{1 \leq i_{1}<\cdots<i_{\xi} \leq m}\left(1-\sum_{j=1}^{\xi} w_{i_{j}}\right)\left(\prod_{j=1}^{\xi} \theta(\widetilde{a})\right)^{1 / \xi}\right), \bigcup_{\gamma(\widetilde{a}) \in h(\widetilde{a})}\left\{1-\left(\prod_{1 \leq i_{1}<\ldots<i_{\xi} \leq m}\left(1-\left(\prod_{j=1}^{\xi} \gamma(\widetilde{a})\right)^{1 / \xi}\right)^{\left(1-\sum_{j=1}^{\xi} w_{i_{j}}\right)}\right)^{1 / C_{m-1}^{\xi}}\right\}\right\rangle \\
& =\left\langle s_{\left(1 / C_{m-1}^{\xi}\right)}\left(\sum_{1 \leq i_{1}<\cdots<i_{\xi} \leq m}\left(1-\sum_{j=1}^{\xi} w_{i_{j}}\right)\left(\prod_{j=1}^{\xi} \theta(\widetilde{a})\right)^{1 / \xi}\right), \bigcup_{\gamma(\widetilde{a}) \in h(\widetilde{a})}\left\{1-\left(\prod_{1 \leq i_{1}<\ldots<i_{\xi} \leq m}\left(1-\left(\gamma(\widetilde{a})^{\xi}\right)^{1 / \xi}\right)^{\left(1-\sum_{j=1}^{\xi} w_{i_{j}}\right)}\right)^{1 / C_{m-1}^{\xi}}\right\}\right\rangle \\
& =\left\langle s_{\left(1 / C_{m-1}^{\xi}\right)}\left(\sum_{1 \leq i_{1}<\ldots<i \xi \leq m}\left(1-\sum_{j=1}^{\xi} w_{i_{j}}\right)\left(\prod_{j=1}^{\xi} \theta(\widetilde{a})\right)^{1 / \xi}\right), \bigcup_{\gamma(\widetilde{a}) \in h(\widetilde{a})}\left\{1-\left(\prod_{1 \leq i_{1}<\ldots<i_{\xi} \leq m}(1-\gamma(\widetilde{a}))\left(1-\sum_{j=1}^{\xi} w_{i_{j}}\right)\right)^{1 / C_{m-1}^{\xi}}\right\}\right\rangle \\
& \left.=\left\langle s_{\left(1 / C_{m-1}^{\xi}\right)}\right)\left(\sum_{1 \leq i_{1}<\cdots<i_{\xi} \leq m}\left(1-\sum_{j=1}^{\xi} w_{i_{j}}\right)\left(\prod_{j=1}^{\xi} \theta(\widetilde{a})\right)^{1 / \xi}\right), \bigcup_{\gamma(\widetilde{a}) \in h(\widetilde{a})}\left\{1-\left((1-\gamma(\widetilde{a}))^{C_{m}^{\xi}-} \prod_{1 \leq i_{1}<\cdots<i_{\xi} \leq m}\left(\sum_{j=1}^{\xi} w_{i_{j}}\right)\right)^{1 / C_{m-1}^{\xi}}\right\}\right\rangle \\
& \left.=\left\langle s_{\left(1 / C_{m-1}^{\xi}\right)}\right)\left(\left(C_{m}^{\xi}-\sum_{i=1}^{m} C_{m-1}^{\xi-1} w_{i}\right) \theta(\widetilde{a})\right), \bigcup_{\gamma(\widetilde{a}) \in h(\widetilde{a})}\left\{1-\left((1-\gamma(\widetilde{a}))^{C_{m}^{\xi}-\sum_{i=1}^{m} C_{m-1}^{\xi-1} w_{i}}\right)^{1 / C_{m-1}^{\xi}}\right\}\right\rangle \\
& \left.=\left\langle s_{\left(1 / C_{m-1}^{\xi}\right.}\right)\left(\left(C_{m}^{\xi}-C_{m-1}^{\xi-1} \sum_{i=1}^{m} w_{i}\right) \theta(\widetilde{a})\right), \bigcup_{\gamma(\tilde{a}) \in h(\widetilde{a})}\left\{1-\left((1-\gamma(\widetilde{a}))^{C_{m}^{\xi}-C_{m-1}^{\xi-1} \sum_{i=1}^{m} w_{i}}\right)^{1 / C_{m-1}^{\xi}}\right\}\right\rangle .
\end{aligned}
$$

Due to $\sum_{i=1}^{m} w_{i}=1$, we can obtain

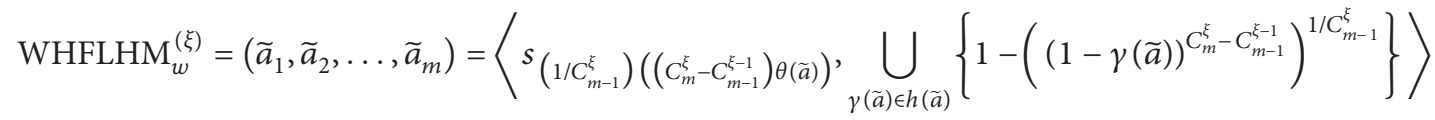

$$
\begin{aligned}
& =\left\langle s_{(\xi !(m-1-\xi) ! !(m-1) !)((m-1) ! ! \xi ! !(m-1-\xi) !) \theta(\widetilde{a})}, \bigcup_{\gamma(\widetilde{a}) \in h(\widetilde{a})}\left\{1-\left((1-\gamma(\widetilde{a}))^{((m-1) ! ! \xi !(m-1-\xi) !)}\right)^{(\xi !(m-1-\xi) ! /(m-1) !)}\right\}\right\rangle \\
& =\left\langle s_{\theta(\tilde{a})}, h(\widetilde{a})\right\rangle=\tilde{a} .
\end{aligned}
$$

(2) For the second situation, when $\xi=m$, we have

$$
\begin{aligned}
& \operatorname{WHFLHM}_{w}^{(\xi)}\left(\widetilde{a}_{1}, \widetilde{a}_{2}, \ldots, \widetilde{a}_{m}\right)=\left\langle s \prod_{j=1}^{\xi} \theta\left(\widetilde{a}_{i}\right)^{\left(1-w_{i}\right) /(m-1)}, \bigcup_{\gamma(\widetilde{a}) \in h(\widetilde{a})}\left\{\prod_{j=1}^{\xi}\left(\gamma\left(\widetilde{a}_{i}\right)\right)^{\left(1-w_{i}\right) /(m-1)}\right\}\right\rangle \\
& =\left\langle s_{\theta\left(\widetilde{a}_{i}\right)}\left(\left(m-\sum_{j=1}^{m} w_{i}\right) /(m-1)\right), \partial \bigcup_{\gamma(\widetilde{a}) \in h(\widetilde{a})}\left\{(\gamma(\widetilde{a}))\left(\left(m-\sum_{j=1}^{m} w_{i}\right) /(m-1)\right)\right\}\right\rangle .
\end{aligned}
$$


Due to $\sum_{i=1}^{m} w_{i}=1$, we can obtain

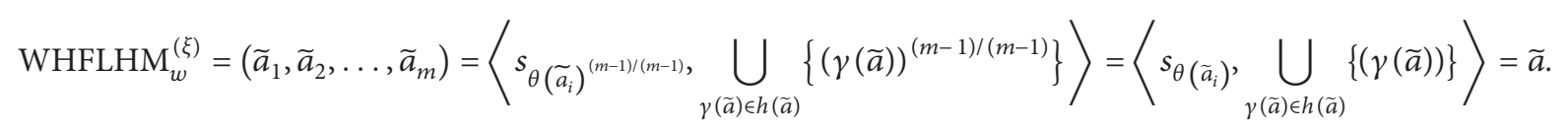

Theorem $8 \quad$ (monotonicity). Let $\mathscr{A}=\left\{\widetilde{a}_{i}=\left\langle s_{\theta\left(\tilde{a}_{i}\right)}, h_{\mathscr{A}}\right.\right.$ $\left.\left.\left(\tilde{a}_{i}\right)\right\rangle \mid i=1,2, \ldots, m\right\}$ and $\mathscr{B}=\left\{\widetilde{b}_{i}=\left\langle s_{\theta\left(\tilde{b}_{i}\right)}, h_{\mathscr{B}}\left(\tilde{a}_{i}\right)\right\rangle \mid i=\right.$ $1,2, \ldots, m\}$ be two sets of HFLEs, and $w=\left\{w_{1}, w_{2}, \ldots, w_{m}\right\}^{T}$ is the associated weight vector of $\widetilde{a}_{i}(i=1,2, \ldots, m)$ such that $w_{i} \in[0,1]$ and $\sum_{i=1}^{m} w_{i}=1$. If $\theta\left(\widetilde{a}_{i}\right) \geq \theta\left(\widetilde{b}_{i}\right)$ and $h_{\mathscr{A}}\left(\widetilde{a}_{i}\right) \geq h_{\mathscr{B}}\left(\widetilde{b}_{i}\right)$ for each $i$, then $\operatorname{WHFLHM}_{w}^{(\xi)}\left(\widetilde{a}_{1}, \widetilde{a}_{2}, \ldots, \widetilde{a}_{m}\right) \geq \operatorname{WHFLHM}_{w}^{(\xi)}\left(\widetilde{b}_{1}, \widetilde{b}_{2}, \ldots, \widetilde{b}_{m}\right)$.
Proof. Since $\xi \geq 1, \theta\left(\widetilde{a}_{i_{j}}\right) \geq \theta\left(\widetilde{b}_{i_{j}}\right) \geq 0$, we have

$$
\prod_{j=1}^{\xi} \theta\left(\tilde{a}_{i_{j}}\right) \geq \prod_{j=1}^{\xi} \theta\left(\widetilde{b}_{i_{j}}\right),\left(\prod_{j=1}^{\xi} \theta\left(\tilde{a}_{i_{j}}\right)\right)^{1 / \xi} \geq\left(\prod_{j=1}^{\xi} \theta\left(\widetilde{b}_{i_{j}}\right)\right)^{1 / \xi}
$$

Then,

$$
\underset{1 \leq i_{1}<\cdots<i_{\xi} \leq m}{\bigoplus}\left(1-\sum_{j=1}^{\xi} w_{i_{j}}\right)\left(\prod_{j=1}^{\xi} \theta\left(\tilde{a}_{i_{j}}\right)\right)^{1 / \xi} \geq \underset{1 \leq i_{1}<\cdots<i_{\xi} \leq m}{\bigoplus_{j=1}}\left(1-\sum_{j=1}^{\xi} w_{i_{j}}\right)\left(\prod_{j=1}^{\xi} \theta\left(\widetilde{b}_{i_{j}}\right)\right)^{1 / \xi}
$$

Accordingly,

$$
\frac{1}{C_{m-1}^{\xi}}\left(\underset{1 \leq i_{1}<\cdots<i_{\xi} \leq m}{\bigoplus_{j=1}}\left(1-\sum_{j=1}^{\xi} w_{i_{j}}\right)\left(\prod_{j=1}^{\xi} \theta\left(\tilde{a}_{i_{j}}\right)\right)^{1 / \xi}\right) \geq \frac{1}{C_{m-1}^{\xi}}\left(\underset{1 \leq i_{1}<\cdots<i_{\xi} \leq m}{\bigoplus_{j=1}}\left(1-\sum_{i_{j}}^{\xi} w_{i_{j}}\right)\left(\prod_{j=1}^{\xi} \theta\left(\widetilde{b}_{i_{j}}\right)\right)^{1 / \xi}\right) .
$$

That is, $s_{\theta\left(\tilde{a}_{i}\right)} \geq s_{\theta\left(\tilde{b}_{i}\right)}$.

Then,

Since $h\left(\widetilde{a}_{i_{j}}\right) \geq h\left(\widetilde{b}_{i_{j}}\right)$ and $\gamma\left(\widetilde{a}_{i_{j}}\right) \geq \gamma\left(\widetilde{b}_{i_{j}}\right)$, we have

$$
\prod_{j=1}^{\xi} \gamma\left(\tilde{a}_{i_{j}}\right) \geq \prod_{j=1}^{\xi} \gamma\left(\widetilde{b}_{i_{j}}\right), 1-\left(\prod_{j=1}^{\xi} \gamma\left(\tilde{a}_{i_{j}}\right)\right)^{1 / \xi} \leq 1-\left(\prod_{j=1}^{\xi} \gamma\left(\widetilde{b}_{i_{j}}\right)\right)^{1 / \xi} .
$$

$$
\prod_{1 \leq i_{1}<\ldots<i_{\xi} \leq m}\left(1-\left(\prod_{j=1}^{\xi} \gamma\left(\tilde{a}_{i_{j}}\right)\right)^{1 / \xi}\right)^{\left(1-\sum_{j=1}^{\xi} w_{i_{j}}\right)} \leq \prod_{1 \leq i_{1}<\ldots<i_{\xi} \leq m}\left(1-\left(\prod_{j=1}^{\xi} \gamma\left(\widetilde{b}_{i_{j}}\right)\right)^{1 / \xi}\right)^{\left(1-\sum_{j=1}^{\xi} w_{i_{j}}\right)} .
$$


Therefore,

$$
1-\left(\prod_{1 \leq i_{1}<\cdots<i_{\xi} \leq m}\left(1-\left(\prod_{j=1}^{\xi} \gamma\left(\tilde{a}_{i_{j}}\right)\right)^{1 / \xi}\right)^{\left(1-\sum_{j=1}^{\xi} w_{i_{j}}\right)}\right)^{1 / C_{m-1}^{\xi}} \geq 1-\left(\prod_{1 \leq i_{1}<\ldots<i_{\xi} \leq m}\left(1-\left(\prod_{j=1}^{\xi} \gamma\left(\tilde{b}_{i_{j}}\right)\right)^{1 / \xi}\right)^{\left(1-\sum_{j=1}^{\xi} w_{i_{j}}\right)}\right)^{1 / C_{m-1}^{\xi}} .
$$

Besides, let $\quad s(\widetilde{a})=\left((1 / \sharp h) \sum_{\gamma \in h} \gamma\right) s_{\theta(\tilde{a})} \quad$ and $s(\tilde{b})=\left((1 / \sharp h) \sum_{\gamma \in h} \gamma\right) s_{\theta(\tilde{b})}$ be the score function of $\tilde{a}$ and $\widetilde{b}$, respectively. Based on the above analysis, we have $s(\widetilde{a}) \geq s(\tilde{b})$. That is, $\operatorname{WHFLHM}^{(\xi)}\left(\widetilde{a}_{1}, \quad \tilde{a}_{2}, \ldots, \tilde{a}_{m}\right) \geq$ $\operatorname{WHFLHM}^{(\xi)}\left(\tilde{b}_{1}, \widetilde{b}_{2}, \ldots, \tilde{b}_{m}\right)$.

Theorem 9 (boundedness). Let $\mathscr{A}=\left\{\widetilde{a}_{i}=\left\langle s_{\theta\left(\tilde{a}_{i}\right)}, h_{\mathscr{A}}\right.\right.$ $\left.\left.\left(\tilde{a}_{i}\right)\right\rangle \mid i=1,2, \ldots, m\right\}$ be a collection of HFLEs, and weight vector $w_{i} \in[0,1], \sum_{i=1}^{m} w_{i}=1$. Assume that $\widetilde{a}^{-}=\min \left(\widetilde{a}_{i}, \widetilde{a}_{2}\right.$, $\left.\ldots, \widetilde{a}_{m}\right)$ and $\tilde{a}^{+}=\max \left(\widetilde{a}_{i}, \widetilde{a}_{2}, \ldots, \widetilde{a}_{m}\right)$, then

$$
\tilde{a}^{-} \leq \operatorname{WHFLHM}_{w}^{(\xi)}\left(\tilde{a}_{1}, \tilde{a}_{2}, \ldots, \tilde{a}_{m}\right) \leq \tilde{a}^{+} .
$$

Proof. According to Theorem 8, we have

$$
\begin{array}{r}
\operatorname{WHFLHM}_{w}^{(\xi)}\left(\tilde{a}^{-}, \tilde{a}^{-}, \ldots, \tilde{a}^{-}\right) \leq \operatorname{WHFLHM}^{(\xi)}\left(\widetilde{a}_{1}, \widetilde{a}_{2}, \ldots, \widetilde{a}_{m}\right) \\
\leq \operatorname{HFLHM}^{\xi}\left(\tilde{a}^{+}, \tilde{a}^{+}, \ldots, \tilde{a}^{+}\right) .
\end{array}
$$

According to Theorem 7, we have

$$
\begin{gathered}
\operatorname{WHFLHM}_{w}^{(\xi)}\left(\tilde{a}^{-}, \tilde{a}^{-}, \ldots, \tilde{a}^{-}\right)=\tilde{a}^{-}, \\
\operatorname{WHFLHM}^{(\xi)}\left(\tilde{a}^{+}, \tilde{a}^{+}, \ldots, \tilde{a}^{+}\right)=\tilde{a}^{+} .
\end{gathered}
$$

Hence, we obtain

$$
\tilde{a}^{-} \leq \operatorname{WHFLHM}_{w}^{(\xi)}\left(\tilde{a}_{1}, \tilde{a}_{2}, \ldots, \tilde{a}_{m}\right) \leq \tilde{a}^{+} .
$$

Theorem 10 (commutativity). Let $\tilde{a}_{i}=\left\langle s_{\theta\left(\tilde{a}_{i}\right)}, h\left(\widetilde{a}_{i}\right)\right\rangle(i=$ $1,2, \ldots, m)$ and $\tilde{b}_{i}=\left\langle s_{\theta\left(\tilde{a}_{i}\right)}, h\left(\tilde{b}_{i}\right)\right\rangle(i=1,2, \ldots, m)$ be $a$ collection of HFLEs, and $w_{i} \in[0,1], \sum_{i=1}^{m} w_{i}=1$. Assume that $\left(\tilde{b}_{1}, \tilde{b}_{2}, \ldots, \tilde{b}_{m}\right)$ is any permutation of $\left(\tilde{a}_{1}, \tilde{a}_{2}, \ldots, \tilde{a}_{m}\right)$, then

$$
\operatorname{WHFLHM}_{w}^{(\xi)}\left(\widetilde{a}_{1}, \widetilde{a}_{2}, \ldots, \widetilde{a}_{m}\right)=\operatorname{WHFLHM}_{w}^{(\xi)}\left(\widetilde{b}_{1}, \widetilde{b}_{2}, \ldots, \widetilde{b}_{m}\right) .
$$

Proof. Since $\left(\widetilde{b}_{1}, \widetilde{b}_{2}, \ldots, \widetilde{b}_{m}\right)$ is any permutation of $\left(\tilde{a}_{1}, \widetilde{a}_{2}, \ldots, \tilde{a}_{m}\right)$, then

$$
\begin{aligned}
& \frac{1}{C_{m-1}^{\xi}}\left(\underset{1 \leq i_{1}<\cdots<i_{\xi} \leq m}{\bigoplus}\left(1-\sum_{j=1}^{\xi} w_{i_{j}}\right)\left(\bigotimes_{j=1}^{\xi} \widetilde{a}_{i_{j}}\right)^{1 / \xi}\right)=\frac{1}{C_{m-1}^{\xi}}\left(\underset{1 \leq i_{1}<\cdots<i_{\xi} \leq m}{\bigoplus_{j=1}}\left(1-\sum_{i_{j}}^{\xi} w_{i}\right)\left(\bigotimes_{j=1}^{\xi} \widetilde{b}_{i_{j}}\right)^{1 / \xi}\right)(1 \leq \xi \leq m), \\
& \bigotimes_{i=1}^{\xi} \tilde{a}_{i}^{\left(1-w_{i}\right) /(m-1)}=\bigotimes_{i=1}^{\xi} \tilde{b}_{i}^{\left(1-w_{i}\right) /(m-1)}(\xi=m) .
\end{aligned}
$$

Accordingly, $\quad \operatorname{WHFLHM}_{w}^{(\xi)}\left(\widetilde{a}_{1}, \tilde{a}_{2}, \ldots, \tilde{a}_{m}\right)=$ $\operatorname{WHFLHM}_{w}^{(\xi)}\left(\widetilde{b}_{1}, \widetilde{b}_{2}, \ldots, \widetilde{b}_{m}\right)$.

In the following, we shall discuss several particular cases of the HFLHM operator obtained by assigning different values of parameter $\xi$.
Case 3. When $\xi=1$, then the $\mathrm{WHFLHM}_{w}^{(\xi)}$ operator will degenerate as

$$
\begin{aligned}
\operatorname{WHFLHM}_{w}^{(1)} & =\left(\widetilde{a}_{1}, \tilde{a}_{2}, \ldots, \tilde{a}_{m}\right)=\frac{1}{C_{m-1}^{1}}\left(\underset{1 \leq i_{1}<\ldots<i_{\xi} \leq m}{\bigoplus_{j=1}}\left(1-\sum_{j=1}^{1} w_{i_{j}}\right)\left(\bigotimes_{j=1}^{1} \tilde{a}_{i_{j}}\right)^{1 / 1}\right) \\
& =\left\langle s_{(1 /(m-1))}\left(\sum_{1 \leq i_{1} \leq m}\left(1-w_{i}\right) \theta\left(\tilde{a}_{i}\right)\right)^{\prime} \underset{\gamma\left(\tilde{a}_{i}\right) \in h\left(\tilde{a}_{i}\right)(i=1,2, \ldots, m)}{ }\left\{1-\left(\prod_{1 \leq i_{1} \leq m}\left(1-\gamma\left(\tilde{a}_{i}\right)\right)^{\left(1-w_{i}\right)}\right)^{1 / m-1}\right\}\right\rangle .
\end{aligned}
$$


Case 4. When $\xi=m$, then the $\operatorname{WHFLHM}_{w}^{(\xi)}$ operator will degenerate as

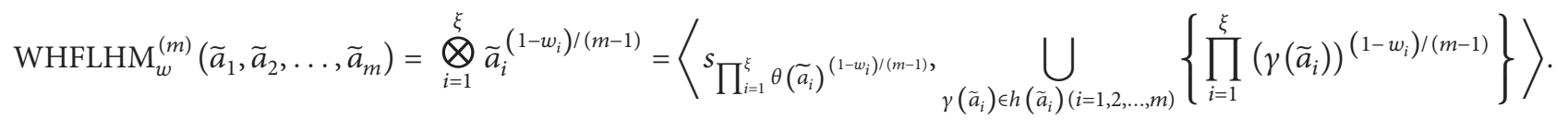

Example 3. Let $\widehat{a}_{1}=\left\langle s_{2},\{0.3,0.4\}\right\rangle, \widehat{a}_{2}=\left\langle s_{5},\{0.6,0.7\}\right\rangle$, and $\widehat{a}_{3}=\left\langle s_{6},\{0.8,0.9\}\right\rangle$ be three HFLEs, and the weighting vector be $w=(0.2,0.3,0.5)^{T}$, then the aggregation value of them can be obtained by the WHFLHM operator, which is shown as follows (suppose $\xi=2$ ):

$$
\begin{aligned}
& \operatorname{WHFLHM}_{w}^{(\xi)}=\left(\widetilde{a}_{1}, \widetilde{a}_{2}, \ldots, \widetilde{a}_{m}\right)=\frac{1}{C_{m-1}^{\xi}}\left(\underset{1 \leq i_{1}<\cdots<i_{\xi} \leq m}{\bigoplus}\left(1-\sum_{j=1}^{\xi} w_{i_{j}}\right)\left(\bigotimes_{j=1}^{\xi} \widetilde{a}_{i_{j}}\right)^{1 / \xi}\right) \\
& =\left\langle S\left(1 / C_{m-1}^{\xi}\right)\left(\sum_{1 \leq i_{1}<\cdots<i_{\xi} \leq m}\left(1-\sum_{j=1}^{\xi} w_{i_{j}}\right)\left(\prod_{j=1}^{\xi} \theta\left(\tilde{a}_{i_{j}}\right)\right)^{1 / \xi}\right)^{\prime}\right. \\
& \cdot\left(\bigcup_{\gamma\left(\tilde{a}_{i}\right) \in h\left(\tilde{a}_{i}\right)(i=1,2, \ldots, m)}\left\{1-\left(\prod_{1 \leq i_{1}<\ldots<i_{\xi} \leq m}\left(1-\left(\prod_{j=1}^{\xi} \gamma\left(\tilde{a}_{i_{j}}\right)\right)^{1 / \xi}\right)^{\left(1-\sum_{j=1}^{\xi} w_{i_{j}}\right)}\right)^{1 / C_{m-1}^{\xi}}\right\}\right) \\
& =\left\langle s_{1.2386},\{0.5103,0.5330,0.5436,0.5692,0.5608,0.5839,0.5945,0.6199\}\right\rangle \text {. }
\end{aligned}
$$

\section{Hesitant Fuzzy Linguistic Dual Hamy Mean Operators}

In the theory research of aggregation operators, there are two kinds of operator including the original operator and its dual operator, for instance, the arithmetic average operator and the geometric operator. So, we will investigate the DHM operator under HFLS setting.

\subsection{HFLDHM Operator}

Definition 11 (see [56]). Let $\mathscr{A}=\left\{\widetilde{a}_{i}=\left\langle s_{\theta\left(\tilde{a}_{i}\right)}, h_{\mathscr{A}}\left(\tilde{a}_{i}\right)\right\rangle \mid i=\right.$ $1,2, \ldots, m\}$ be a set of HFLEs, then the HFLDHM operator is depicted as

$$
\operatorname{HFLDHM}^{(\xi)}\left(\tilde{a}_{1}, \tilde{a}_{2}, \ldots, \tilde{a}_{m}\right)=\left(\bigotimes_{1 \leq i_{1}<\cdots<i_{\xi} \leq m}\left(\frac{\bigoplus_{j=1}^{\xi} \tilde{a}_{i_{j}}}{\xi}\right)\right)^{1 / C_{m}^{\xi}}
$$

where $\xi$ is a parameter, $\xi=1,2, \ldots, m, i_{1}, i_{2}, \ldots, i_{\xi}$ are $\xi$ integer values taken from the set $\{1,2, \ldots, m\}$ of $m$ integer values, $C_{m}^{\xi}$ is the binomial coefficient, and $C_{m}^{\xi}=(m ! /$ $\xi !(m-\xi) !)$.

Theorem 11. Let $\mathscr{A}=\left\{\widetilde{a}_{i}=\left\langle s_{\theta\left(\tilde{a}_{i}\right)}, h_{\mathscr{A}}\left(\widetilde{a}_{i}\right)\right\rangle \mid i=1,2, \ldots, m\right\}$ be a collection of HFLEs, then the fused value obtained by the HFLHM operator is also a HFLE, and it can be expressed as

$$
\begin{aligned}
& \operatorname{HFLDHM}^{(\xi)}\left(\tilde{a}_{1}, \tilde{a}_{2}, \ldots, \tilde{a}_{m}\right)=\left(\bigotimes_{1 \leq i_{1}<\ldots<i_{\xi} \leq m}\left(\frac{\bigoplus_{j=1}^{\xi} \tilde{a}_{i_{j}}}{\xi}\right)\right)^{1 / C_{m}^{\xi}} \\
& =\left\langle s \prod_{\left(\prod_{1 \leq i_{1}<\cdots<i_{\xi} \leq m}\left(\sum_{j=1}^{\xi} \tilde{a}_{i_{j}} / \xi\right)\right)^{1 / C_{m}^{\xi}},} \bigcup_{\gamma\left(\tilde{a}_{i}\right) \in h\left(\tilde{a}_{i}\right)(i=1,2, \ldots, m)}\left(\prod_{1 \leq i_{1}<\ldots<i_{\xi} \leq m}\left(1-\left(\prod_{j=1}^{\xi}\left(1-\tilde{a}_{i_{j}}\right)\right)^{1 / \xi}\right)\right)^{1 / C_{m}^{\xi}}\right\rangle .
\end{aligned}
$$


The proof of Theorem 11 is analogue to Theorem 1, so we omit it here.

Similar to the HFLHM operator, the following properties of the HFLDHM operator are investigated.

Theorem 12 (idempotency). Let $\mathscr{A}=\left\{\tilde{a}_{i}=\left\langle s_{\theta\left(\tilde{a}_{i}\right)}, h_{\mathscr{A}}\right.\right.$ $\left.\left.\left(\tilde{a}_{i}\right)\right\rangle \mid i=1,2, \ldots, m\right\}$ be a collection of HFLEs; if $\tilde{a}=\tilde{a}=\left\langle s_{\theta\left(\tilde{a}_{i}\right)}, h_{\mathscr{A}}\left(\tilde{a}_{i}\right)\right\rangle(i=1,2, \ldots, m)$, then

$$
\operatorname{HFLDHM}^{(\xi)}\left(\widetilde{a}_{1}, \tilde{a}_{2}, \ldots, \tilde{a}_{m}\right)=\tilde{a} .
$$

Theorem 13 (monotonicity). Let $\mathscr{A}=\left\{\tilde{a}_{i}=\left\langle s_{\theta\left(\tilde{a}_{i}\right)}, h_{\mathscr{A}}\right.\right.$ $\left.\left.\left(\tilde{a}_{i}\right)\right\rangle \mid i=1,2, \ldots, m\right\} \quad$ and $\mathscr{A}=\left\{\tilde{a}_{i}=\left\langle s_{\theta\left(\tilde{a}_{i}\right)}, h_{\mathscr{A}}(\tilde{b})_{i}\right\rangle \mid i=\right.$ $1,2, \ldots, m\}$ be two sets of HFLEs; if $\theta\left(\widetilde{a}_{i}\right) \geq \theta\left(\widetilde{b}_{i}\right)$ and $h_{\mathscr{A}}\left(\widetilde{a}_{i}\right) \geq h_{\mathscr{B}}\left(\tilde{b}_{i}\right)$ for each $i(i=1,2, \ldots, m)$, then

$$
\operatorname{HFLDHM}^{(\xi)}\left(\widetilde{a}_{1}, \tilde{a}_{2}, \ldots, \tilde{a}_{m}\right) \geq \operatorname{HFLDHM}^{(\xi)}\left(\widetilde{b}_{1}, \tilde{b}_{2}, \ldots, \widetilde{b}_{m}\right) \text {. }
$$

Theorem 14 (boundedness). Let $\mathscr{A}=\left\{\tilde{a}_{i}=\left\langle s_{\theta\left(\tilde{a}_{i}\right)}, h_{\mathscr{A}}\right.\right.$ $\left.\left.\left(\tilde{a}_{i}\right)\right\rangle \mid i=1,2, \ldots, m\right\}$ be a collection of HFLEs. Assume that $\tilde{a}^{-}=\min \left(\tilde{a}_{i}, \tilde{a}_{2}, \ldots, \tilde{a}_{m}\right)$ and $\tilde{a}^{+}=\max \left(\tilde{a}_{i}, \tilde{a}_{2}, \ldots, \tilde{a}_{m}\right)$, then

$$
\tilde{a}^{-} \leq \operatorname{HFLDHM}^{(\xi)}\left(\tilde{a}_{1}, \tilde{a}_{2}, \ldots, \tilde{a}_{m}\right) \leq \tilde{a}^{+} .
$$

Theorem 15 (commutativity). Let $\tilde{a}_{i}=\left\langle s_{\theta\left(\tilde{a}_{i}\right)}, h\left(\widetilde{a}_{i}\right)\right\rangle \quad(i=$ $1,2, \ldots, m)$ and $\tilde{b}_{i}=\left\langle s_{\theta\left(\tilde{a}_{i}\right)}, h\left(\widetilde{b}_{i}\right)\right\rangle(i=1,2, \ldots, m)$ be two collections of HFLEs. Assume that $\left(\tilde{b}_{1}, \tilde{b}_{2}, \ldots, \widetilde{b}_{m}\right)$ is any permutation of $\left(\widetilde{a}_{1}, \tilde{a}_{2}, \ldots, \widetilde{a}_{m}\right)$, then

$$
\operatorname{HFLDHM}^{(\xi)}\left(\tilde{a}_{1}, \tilde{a}_{2}, \ldots, \tilde{a}_{m}\right)=\operatorname{HFLDHM}^{(\xi)}\left(\tilde{b}_{1}, \tilde{b}_{2}, \ldots, \tilde{b}_{m}\right) \text {. }
$$

\subsection{Hesitant Fuzzy Linguistic Weighted Dual Hamy Mean Operator}

Definition 12. Let $\mathscr{A}=\left\{\widetilde{a}_{i}=\left\langle s_{\theta\left(\tilde{a}_{i}\right)}, h_{\mathscr{A}}\left(\tilde{a}_{i}\right)\right\rangle \mid i=1,2\right.$, $\ldots, m\}$ be a collection of HFLEs, and $w=\left\{w_{1}, w_{2}, \ldots, w_{n}\right\}^{T}$ is the associated weight vector of $\widetilde{a}_{i}(i=1,2, \ldots, m)$ such that $w_{i} \in[0,1]$ and $\sum_{i=1}^{n} w_{i}=1$. Then, the WHFLDHM operator is described as follows:

$$
\operatorname{WHFLDHM}_{w}^{(\xi)}\left(\tilde{a}_{1}, \tilde{a}_{2}, \ldots, \tilde{a}_{m}\right)= \begin{cases}\left(\bigotimes_{1 \leq i_{1}<\cdots<i_{\xi} \leq m}\left(1-\sum_{j=1}^{\xi} w_{i_{j}}\right) \frac{\bigoplus_{j=1}^{\xi} \tilde{a}_{i_{j}}}{\xi}\right)^{1 / C_{m-1}^{\xi}}, & 1 \leq \xi<m, \\ \bigoplus_{i=1}^{\xi} \tilde{a}_{i}^{\left(1-w_{i}\right) /(m-1)}, & \xi=m,\end{cases}
$$

where $\xi$ is a parameter, $\xi=1,2, \ldots, m, i_{1}, i_{2}, \ldots, i_{\xi}$ are $\xi$ integer values taken from the set $\{1,2, \ldots, m\}$ of $m$ integer values, $C_{m}^{\xi}$ is the binomial coefficient, and $C_{m}^{\xi}=(m ! / \xi !(m-\xi) !)$.
Theorem 16. Let $\mathscr{A}=\left\{\tilde{a}_{i}=\left\langle s_{\theta\left(\tilde{a}_{i}\right)}, h_{\mathscr{A}}\left(\tilde{a}_{i}\right)\right\rangle \mid i=1,2, \ldots, m\right\}$ be a collection of HFLEs, and the associated weight vector $w_{i} \in[0,1]$ and $\sum_{i=1}^{n} w_{i}=1$. Then, the aggregation result obtained by Definition 12 is also a HFLE, and it can be expressed as

$$
\begin{aligned}
\operatorname{WHFLDHM}_{w}^{(\xi)}\left(\tilde{a}_{1}, \tilde{a}_{2}, \ldots, \tilde{a}_{m}\right)= & \left(\bigotimes_{1 \leq i_{1}<\cdots<i_{\xi} \leq m}\left(1-\sum_{j=1}^{\xi} w_{i_{j}}\right)\left(\frac{\bigoplus_{j=1}^{\xi} \tilde{a}_{i_{j}}}{\xi}\right)\right)^{1 / C_{m-1}^{\xi}} \\
= & \left\langle\int_{\left(\prod_{1 \leq i_{1}<\ldots<i_{\xi} \leq m}\left(1-\sum_{j=1}^{\xi} w_{i_{j}}\right)\left(\sum_{j=1}^{\xi} \tilde{a}_{i_{j}} / \xi\right)\right)^{1 / C_{m-1}^{\xi},}}\right. \\
& \cdot \bigcup_{\gamma\left(\tilde{a}_{i}\right) \in h\left(\tilde{a}_{i}\right)(i=1,2, \ldots, m)}\left(\prod_{1 \leq i_{1}<\cdots<i_{\xi} \leq m}\left(1-\left(\prod_{j=1}^{\xi}\left(1-\gamma\left(\tilde{a}_{i_{j}}\right)\right)^{1 / \xi}\right)^{\left.\left.\left(1-\sum_{j=1}^{\xi} w_{i_{j}}\right)\right)^{1 / C_{m-1}^{\xi}}\right)}\right\rangle,\right.
\end{aligned}
$$




$$
\begin{aligned}
& \operatorname{WHFLDHM}_{w}^{(\xi)}\left(\widetilde{a}_{1}, \tilde{a}_{2}, \ldots, \tilde{a}_{m}\right)=\bigoplus_{i=1}^{\xi} \tilde{a}_{i}^{\left(1-w_{i}\right) /(m-1)}
\end{aligned}
$$

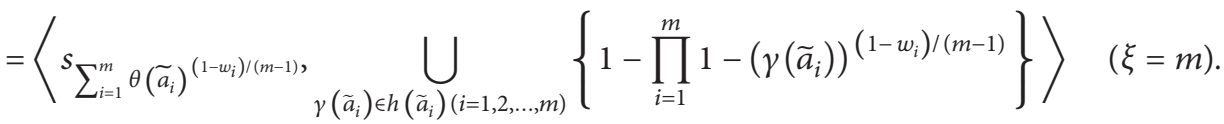

The proof of Theorem 16 is analogue to Theorem 6, so we omit it here.

Similar to the HFLHM operator, the following properties of the WHFLDHM operator are investigated.

Theorem 17 (idempotency). Let $\mathscr{A}=\left\{\tilde{a}_{i}=\left\langle s_{\theta\left(\tilde{a}_{i}\right)}, h_{\mathscr{A}}\right.\right.$ $\left.\left.\left(\tilde{a}_{i}\right)\right\rangle \mid i=1,2, \ldots, m\right\}$ be a collection of HFLEs, and $w=\left\{w_{1}, w_{2}, \ldots, w_{m}\right\}^{T}$ is the associated weight vector of $\tilde{a}_{i}(i=1,2, \ldots, m)$ such that $w_{i} \in[0,1]$ and $\sum_{i=1}^{m} w_{i}=1$. If $\tilde{a}_{i}=\tilde{a}=\left\langle s_{\theta(\tilde{a})}, h_{\mathscr{A}}(\widetilde{a})\right\rangle(i=1,2, \ldots, m)$, then

$$
\operatorname{WHFLDHM}_{w}^{(\xi)}\left(\widetilde{a}_{1}, \tilde{a}_{2}, \ldots, \widetilde{a}_{m}\right)=\tilde{a} .
$$

Theorem 18 (monotonicity). Let $\mathscr{A}=\left\{\tilde{a}_{i}=\left\langle s_{\theta\left(\tilde{a}_{i}\right)}, h_{\mathscr{A}}\right.\right.$ $\left.\left.\left(\tilde{a}_{i}\right)\right\rangle \mid i=1,2, \ldots, m\right\}$ and $\mathscr{B}=\left\{\tilde{a}_{i}=\left\langle s_{\theta\left(\tilde{a}_{i}\right)}, h_{\mathscr{A}}\left(\tilde{b}_{i}\right)\right\rangle \mid i=\right.$ $1,2, \ldots, m\}$ be two sets of HFLEs, and $w=\left\{w_{1}, w_{2}, \ldots, w_{m}\right\}^{T}$ is the associated weight vector of $\tilde{a}_{i}(i=1,2, \ldots, m)$ such that $w_{i} \in[0,1]$ and $\sum_{i=1}^{m} w_{i}=1$. If $\theta\left(\widetilde{a}_{i}\right) \geq \theta\left(\widetilde{b}_{i}\right)$ and $h\left(\widetilde{a}_{i}\right) \geq h\left(\widetilde{b}_{i}\right)$ for each $i(i=1,2, \ldots, m)$, then

$\operatorname{WHFLDHM}_{w}^{(\xi)}\left(\widetilde{a}_{1}, \widetilde{a}_{2}, \ldots, \widetilde{a}_{m}\right) \geq \operatorname{WHFLDHM}_{w}^{(\xi)}\left(\widetilde{b}_{1}, \widetilde{b}_{2}, \ldots, \widetilde{b}_{m}\right)$.

Theorem 19 (boundedness). Let $\mathscr{A}=\left\{\tilde{a}_{i}=\left\langle s_{\theta\left(\tilde{a}_{i}\right)}, h_{\mathscr{A}}\right.\right.$ $\left.\left.\left(\tilde{a}_{i}\right)\right\rangle \mid i=1,2, \ldots, m\right\}$ be a collection of HFLEs, and weight vector $w_{i} \in[0,1], \sum_{i=1}^{m} w_{i}=1$. Assume that $\tilde{a}^{-}=\min \left(\tilde{a}_{i}, \widetilde{a}_{2}\right.$, $\left.\ldots, \tilde{a}_{m}\right)$ and then,

$$
\tilde{a}^{-} \leq \operatorname{WHFLDHM}_{w}^{(\xi)}\left(\widetilde{a}_{1}, \tilde{a}_{2}, \ldots, \tilde{a}_{m}\right) \leq \tilde{a}^{+} .
$$

Theorem 20 (commutativity). Let $\tilde{a}_{i}=\left\langle s_{\theta\left(\tilde{a}_{i}\right)}, h\left(\tilde{a}_{i}\right)\right\rangle(i=$ $1,2, \ldots, m)$ and $\widetilde{b}_{i}=\left\langle s_{\theta\left(\tilde{b}_{i}\right)}, h\left(\widetilde{b}_{i}\right)\right\rangle(i=1,2, \ldots, m)$ be two collections of HFLEs. Assume that $\left(\tilde{b}_{1}, \tilde{b}_{2}, \ldots, \tilde{b}_{m}\right)$ is any permutation of $\left(\tilde{a}_{1}, \tilde{a}_{2}, \ldots, \widetilde{a}_{m}\right)$, then

$\operatorname{WHFLDHM}_{w}^{(\xi)}\left(\tilde{a}_{1}, \tilde{a}_{2}, \ldots, \tilde{a}_{m}\right)=\operatorname{WHFLDHM}_{w}^{(\xi)}\left(\widetilde{b}_{1}, \widetilde{b}_{2}, \ldots, \widetilde{b}_{m}\right)$.

\section{A New Approach to MADM Based on the WHFLHM Operator and the WHFLDHM Operator}

In this part, we shall present a novel MADM approach based on the WHFLHM operator and the WHFLDHM operator to solve the decision problem with HFL information.

A classical MADM problem for selecting a best alternative with HFL information is stated as follows. Let $X=$ $\left\{x_{k} \mid k=1,2, \ldots, p\right\}$ be a group of alternatives. $A=\left\{a_{l} \mid l=\right.$ $1,2, \ldots, q\}$ is a collection of attributes with the weight vector $w=\left(w_{1}, w_{2}, \ldots, w_{q}\right)^{T}, \quad w_{l} \in[0,1](l=1,2, \ldots, q)$, and $\sum_{l=1}^{q} w_{l}=1$. The HFL decision matrix is represented as $\widetilde{H}=\left(\widetilde{a}_{k l}\right)_{p \times q}$, in which $\tilde{a}_{k l}=\left\langle s_{\theta\left(a_{k l}\right)}, h\left(\tilde{a}_{k l}\right)\right\rangle$ is a HFLE, which denotes the assessment value of $x_{k}(k=1,2, \ldots, p)$ on $a_{l}(l=1,2, \ldots, q)$. In the following, we will establish a novel algorithm (see algorithm 1) to carry out the HFLMADM problem on the basis of the presented aggregation operators.

\section{Illustration Example}

In this part, we provide an actual application example which is cited from [40] to prove the validity and applicability of the new approach.

A state-owned enterprise engaged in the sale and production of nonferrous metals has set up an overseas affairs investment department composed of investment experts to invest in countries with great potential worldwide to provide sales channels for nonferrous metals in order to expand its business scope. Recently, the investment sector will choose a country with the greatest potential for industrial investment. Through investigation and research, the expert group takes into account five countries as alternative targets, i.e., $\left\{x_{1}, x_{2}, x_{3}, x_{4}, x_{5}\right\}$. Because there are a host of influence factors in the investment problem, after the analysis and discussion of the expert group, the four most influential factors are identified, including $c_{1}$ : resource reserve (for example, mineral reserve and exploration); $c_{2}$ : political policy (for example, policy support); $c_{3}$ : economic (for example, capital reserve strength and economic stability); and $c_{4}$ : infrastructure (for example, transportation and 
Input: the HFL decision matrix $\widetilde{H}=\left(\widetilde{a}_{k l}\right)_{p \times q}$ and the attribute weight vector $w=\left(w_{1}, w_{2}, \ldots, w_{q}\right)^{T}$. Begin

Step 1: normalize the HFLDM $\widetilde{H}=\left(\widetilde{a}_{k l}\right)_{p \times q}$ to $H=\left(a_{k l}\right)_{p \times q}$ by the following method.

$$
a_{k l}=\left\langle s_{\theta\left(a_{k l}\right)}, h\left(a_{k l}\right)\right\rangle= \begin{cases}\tilde{a}_{k l}=\left\langle s_{\theta\left(a_{k l}\right)}, h\left(\tilde{a}_{k l}\right)\right\rangle, & a_{l} \text { is benefit - type, } \\ \left(\widetilde{a}_{k l}\right)^{c}=\left\langle s_{\theta\left(a_{k l}\right)}, 1-h\left(\tilde{a}_{k l}\right)\right\rangle, & a_{l} \text { is cost - type. }\end{cases}
$$

Step 2: compute the comprehensive assessment value $a_{k}$ for alternative $x_{k}$ by

$$
a_{k}=\operatorname{WHFLHM}_{w}^{(x)}\left(a_{k 1}, a_{k 2}, \ldots, a_{k q}\right)
$$

or

$a_{k}=\operatorname{WHFLDHM}_{w}^{(x)}\left(a_{k 1}, a_{k 2}, \ldots, a_{k q}\right)$.

to aggregate the HFL assessment information.

Step 3: compute the score of the comprehensive assessment value $a_{k}$ by Definition 6 . End.

Step 4: ranking the alternatives and obtaining the optimal one(s).

Algorithm 1: For HFLMADM with HFL information.

machinery facilities). The homologous attribute weight vector is $w=(0.2,0.4,0.3,0.1)^{T}$.

Considering the above factors, the expert group gave the evaluation information of each alternative. The LTS $S=\left\{s_{0}, s_{1}, s_{2}, s_{3}, s_{4}, s_{5}, s_{6}\right\}=\{$ very poor, slightly poor, fair, slightly good, good, very good $\}$ is utilized to evaluate alternatives. The assessment information is provided by experts with HFLE, in which $a_{j}\left(x_{i}\right)$ represents the assessment value of $x_{i}$ on the attribute $c_{j}$. Considering the assessment value $a_{j}\left(x_{i}\right)$, experts reach a consensus based on the selected linguistic term and give their opinion by a certainty value in $[0,1]$ based on their knowledge and cognition, which represents the membership degree of $x_{i}$ to the selected linguistic term under the attribute $a_{j}$. After that, all the possible values based on each selected linguistic term are collected together, and the same $\mathrm{MD}$ in a linguistic term only emerge once. Finally, experts reach consensus for the assessment information of all alternatives under considered attributes after discussion and investigation, and the ultimate assessment information matrix expressed by HFLE is determined as shown in Table 1 .

\subsection{Decision-Making Steps}

Step 1. Standardize the HFLDM $\widetilde{H}=\left(\widetilde{a}_{i j}\right)_{m \times n}$.

Since all considered attributes are benefit-type, we need not to standardize the attribute value.

Step 2. Compute comprehensive assessment value $a_{i}$ for alternative $x_{i}$ by the equation in Algorithm 1 and suppose $\xi=2$.

$$
\begin{aligned}
& \tilde{a}_{1}=\left\langle s_{3.5986},\{0.3497,0.3885,0.3938,0.4340,0.3777,0.4184,\right. \\
&0.4225,0.4642\}\rangle ; \\
& \tilde{a}_{2}=\left\langle s_{2.8846},\{0.4704,0.5332,0.5128,0.5758,0.4972,0.5612,\right. \\
&0.5397,0.6032\}\rangle ; \\
& \tilde{a}_{3}=\left\langle s_{3.2061},\{0.5263,0.5488,0.5827,0.6062\}\right\rangle ; \\
& \tilde{a}_{4}=\left\langle s_{2.0165},\{0.5192,0.4695,0.5508,0.6273,0.5806,0.6555\}\right\rangle ; \\
& \tilde{a}_{5}=\left\langle s_{3.8830},\{0.5815,0.6297,0.6021,0.6499,0.6365,0.6851,\right. \\
&0.6566,0.7042\}\rangle .
\end{aligned}
$$

Step 3. Calculate the score of $a_{i}$ by Definition 6 .

$$
\begin{aligned}
& J\left(x_{1}\right)=1.4614, \\
& J\left(x_{2}\right)=1.5481 \\
& J\left(x_{3}\right)=1.8146, \\
& J\left(x_{4}\right)=1.1437 \\
& J\left(x_{5}\right)=2.4975 .
\end{aligned}
$$

Step 4. Rank the alternatives in the descending order, and obtain the best one.

$$
x_{5}>x_{3}>x_{2}>x_{1}>x_{4}
$$

Accordingly, the best alternative is $x_{5}$.

In Step 2, if we utilize the equation in Algorithm 1 to fuse the attribute value, then we obtain

$$
\begin{aligned}
& \tilde{a}_{1}=\left\langle s_{3.6843},\{0.3562,0.4048,0.3974,0.4478,0.3833,0.4327,\right. \\
&0.4236,0.4741\}\rangle ; \\
& \tilde{a}_{2}=\left\langle s_{2.4039},\{0.4810,0.5427,0.5216,0.5797,0.5096,0.5698,\right. \\
&0.5496,0.6053\}\rangle ; \\
& \tilde{a}_{3}=\left\langle s_{3.0992},\{0.5360,0.5648,0.5873,0.6153\}\right\rangle ; \\
& \tilde{a}_{4}=\left\langle s_{1.1635},\{0.5534,0.6275,0.5773,0.6472,0.5773,0.6472\}\right\rangle ; \\
& \tilde{a}_{5}=\left\langle s_{4.1494},\{0.5877,0.6070,0.6402,0.6590,0.6406,0.6585,\right. \\
&0.6899,0.7068\}\rangle .
\end{aligned}
$$

Step 5. Calculate the score of the comprehensive assessment value $a_{i}$ by Definition 6 .

$$
\begin{aligned}
& J\left(x_{1}\right)=1.5289 \\
& J\left(x_{2}\right)=1.3099 \\
& J\left(x_{3}\right)=1.7846 \\
& J\left(x_{4}\right)=0.7039 \\
& J\left(x_{5}\right)=2.6918
\end{aligned}
$$


TABLE 1: The hesitant fuzzy linguistic decision matrix.

\begin{tabular}{lcccc}
\hline Alternative & $a_{1}$ & $a_{2}$ & $a_{3}$ & $a_{4}$ \\
\hline$x_{1}$ & $\left\langle s_{4},\{0.3,0.4\}\right\rangle$ & $\left\langle s_{5},\{0.2,0.4\}\right\rangle$ & $\left\langle s_{4},\{0.5,0.7\}\right\rangle$ & $\left\langle s_{3},\{0.4\}\right\rangle$ \\
$x_{2}$ & $\left\langle s_{2},\{0.5,0.6\}\right\rangle$ & $\left\langle s_{4},\{0.3,0.5\}\right\rangle$ & $\left\langle s_{3},\{0.7\}\right\rangle$ & $\left\langle s_{4},\{0.4,0.6\}\right\rangle$ \\
$x_{3}$ & $\left\langle s_{3},\{0.4,0.6\}\right\rangle$ & $\left\langle s_{4},\{0.4\}\right\rangle$ & $\left\langle s_{6},\{0.7,0.8\}\right\rangle$ & $\left\langle s_{2},\{0.6\}\right\rangle$ \\
$x_{4}$ & $\left\langle s_{2},\{0.3,0.4,0.5\}\right\rangle$ & $\left\langle s_{3},\{0.6\}\right\rangle$ & $\left\langle s_{2},\{0.4,0.7\}\right\rangle$ & $\left\langle s_{2},\{0.8\}\right\rangle$ \\
$x_{5}$ & $\left\langle s_{5},\{0.5,0.7\}\right\rangle$ & $\left\langle s_{5},\{0.5,0.6\}\right\rangle$ & $\left\langle s_{3},\{0.6,0.8\}\right\rangle$ & $\left\langle s_{4},\{0.7\}\right\rangle$ \\
\hline
\end{tabular}

Step 6. Rank the alternatives in the descending order, and obtain the best one.

$$
x_{5}>x_{3}>x_{1}>x_{2}>x_{4} .
$$

Accordingly, the best alternative is $x_{5}$.

6.2. Sensitivity Analysis. The parameter plays a vital role in the process of information integration for ultimate ranking results. Because the WHFLHM operator and the WHFLDHM operator have a changeable parameter, different parameter values reflect different risk preference attitudes of decision makers in solving MADM problems. So, it is indispensable to discuss the influence of different parameters for ranking result. In the next, we take diverse parameter value of $\xi$ in the WHFLHM operator and the WHFLDHM operator to obtain the corresponding ranking results, which are displayed in Tables 2 and 3.

From Tables 2 and 3, the following conclusions for parameter $\xi$ on the decision-making results are summarized:

(1) We can obtain the different score values and decision results by assigning different parameter values of $\xi$. Although the final ordering results of alternatives are slightly different, the best alternative is $x_{5}$. This is because the parameter is taken as different values, and the different correlation of attributes is taken into account. For example, if $\xi=2$, the correlation of two attributes is considered; if $\xi=3$, the inter-relationship of three attributes is considered, which shows that the HM operator has excellent robustness in the issues of information fusion.

(2) The HM operator can reduce to the arithmetic average operator and geometric average operator by taking different parameter value $\xi$. In practice situation, decision makers can analyse the generalization results by some special cases.

(3) When parameters are taken different values, we can get different score values and decision results; it means that the parameter $\xi$ has a good control capability, and different parameters can be regarded as risk preferences of evaluators. Evaluators can choose favourable parameter $\xi$ according to their preferences attitude.

6.3. Comparison Analysis. In order to verify the validity and the remarkable merits of the propounded method, we will address the same demonstrative example by utilizing other approaches including the HFL-TOPSIS method [40], HFL weight average (HFLWA) operator [39], HFL weight geometric (HFLWG) operator, and HFLBM operator. The ranking results and the characteristic comparative with other approaches are manifested in Tables 4 and 5, respectively.

In the next, we will perform a detailed comparative analysis on the basis of the above approaches with our proposed approach. According to our further analysis and the character comparison in Table 5, we obtain the following summarization:

(1) Compared with the approach based on the HFLWA operator proposed by Lin et al. [39], although the HFLWA operator can be utilized to aggregate HFL information, it usually assumes that attributes are independent of each other, and it lacks flexibility and robustness in the course information fusion. In comparison, the proposed approach based on the WHFLHM operator can not only capture the correlation of overall attributes but also can enable the decision process dynamic through a flexible parameter. Moreover, the WHFLHM operator is more universal than the HFLWA operator because it is a particular case of the WHFLHM operator. Accordingly, our developed approach is more appropriate to cope with practical MADM problems.

(2) Compared with HFL-TOPSIS approach in [40], the TOPSIS approach only provides a ranking result of alternatives, and the distance computation of ideal points will lead to information loss phenomenon happening. However, the presented approach on the basis of the WHFLHM operator can directly obtain the comprehensive evaluation value as well as the sorting of alternatives, and the inter-relationship among any number of arbitrary attributes is taken into consideration, i.e., it can reduce information loss to some extent in information integration procedure. At the same time, the adjustable parameter $\xi$ can be assigned different values through evaluator's risk preference. In summary, our developed method is more general and flexible than the HFL-TOPSIS method.

(3) Compared with the HFLWBM operator in [62], the HFLWBM operator can only capture the inter-relation between any two input arguments. However, in the practical application issues, we often need to take into account all arguments provided by the evaluator. The developed HFLWHM and 
TABLE 2: Score value and order relation of alternatives by the WHFLHM operator.

\begin{tabular}{lll}
\hline Parameter & \multicolumn{1}{c}{ Score values of $x_{i}(i=1,2,3,4,5)$} & Ranking order \\
\hline$\xi=1$ & $J\left(x_{1}\right)=1.6598, J\left(x_{2}\right)=1.7974, J\left(x_{3}\right)=2.0881, J\left(x_{4}\right)=1.3914, J\left(x_{5}\right)=2.7724$ & $x_{5}>x_{3}>x_{2}>x_{1}>x_{4}$ \\
$\xi=2$ & $J\left(x_{1}\right)=1.4614, J\left(x_{2}\right)=1.5481, J\left(x_{3}\right)=1.8146, J\left(x_{4}\right)=1.1437, J\left(x_{5}\right)=2.4975$ & $x_{5}>x_{3}>x_{2}>x_{1}>x_{4}$ \\
$\xi=3$ & $J\left(x_{1}\right)=1.5354, J\left(x_{2}\right)=1.6544, J\left(x_{3}\right)=1.8577, J\left(x_{4}\right)=1.2438, J\left(x_{5}\right)=2.6605$ & $x_{5}>x_{3}>x_{2}>x_{1}>x_{4}$ \\
$\xi=4$ & $J\left(x_{1}\right)=1.5157, J\left(x_{2}\right)=1.6327, J\left(x_{3}\right)=1.8283, J\left(x_{4}\right)=1.2339, J\left(x_{5}\right)=2.6433$ & $x_{5}>x_{3}>x_{2}>x_{1}>x_{4}$ \\
\hline
\end{tabular}

TABLE 3: Score value and order relation of alternatives by the WHFLDHM operator.

\begin{tabular}{lll}
\hline Parameter & \multicolumn{1}{c}{ Score values of $x_{i}(i=1,2,3,4,5)$} & Ranking order \\
\hline$\xi=1$ & $J\left(x_{1}\right)=1.1308, J\left(x_{2}\right)=1.1281, J\left(x_{3}\right)=1.5198, J\left(x_{4}\right)=1.0257, J\left(x_{5}\right)=1.8264$ & $x_{5}>x_{3}>x_{1}>x_{2}>x_{4}$ \\
$\xi=2$ & $J\left(x_{1}\right)=1.5289, J\left(x_{2}\right)=1.3099, J\left(x_{3}\right)=1.7846, J\left(x_{4}\right)=0.7039, J\left(x_{5}\right)=2.6918$ & $x_{5}>x_{3}>x_{1}>x_{2}>x_{4}$ \\
$\xi=3$ & $J\left(x_{1}\right)=0.2547, J\left(x_{2}\right)=0.1456, J\left(x_{3}\right)=0.2673, J\left(x_{4}\right)=0.0383, J\left(x_{5}\right)=0.5068$ & $x_{5}>x_{3}>x_{1}>x_{2}>x_{4}$ \\
$\xi=4$ & $J\left(x_{1}\right)=2.9632, J\left(x_{2}\right)=2.4049, J\left(x_{3}\right)=3.1950, J\left(x_{4}\right)=2.3032, J\left(x_{5}\right)=3.7484$ & $x_{5}>x_{3}>x_{1}>x_{2}>x_{4}$ \\
\hline
\end{tabular}

TABle 4: Decision results by utilizing different approaches.

\begin{tabular}{lcr}
\hline Approaches & Parameter value & Order relation \\
\hline HFLWA operator [39] & NO & $x_{5}>x_{3}>x_{2}>x_{1}>x_{4}$ \\
HFLWG operator [39] & NO & $x_{5}>x_{3}>x_{2}>x_{1}>x_{4}$ \\
HFLWPA operator [39] & NO & $x_{5}>x_{3}>x_{2}>x_{1}>x_{4}$ \\
HFL-TOPSIS [40] & NO & $x_{5}>x_{3}>x_{1}>x_{2}>x_{4}$ \\
HFLWBM operator [62] & $p=q=1$ & $x_{5}>x_{3}>x_{1}>x_{2}>x_{4}$ \\
WHFLHM operator & $\xi=2$ & $x_{5}>x_{3}>x_{2}>x_{1}>x_{4}$ \\
WHFLDHM operator & $\xi=2$ & $x_{5}>x_{3}>x_{1}>x_{2}>x_{4}$ \\
\hline
\end{tabular}

TABLE 5: Comparison of characteristics with different MADM approaches.

\begin{tabular}{|c|c|c|c|}
\hline Approaches & $\begin{array}{l}\text { Whether to capture the inter- } \\
\text { relationship between two attributes }\end{array}$ & $\begin{array}{l}\text { Whether to capture the inter- } \\
\text { relationship between multiple attributes }\end{array}$ & $\begin{array}{l}\text { Whether has generalized } \\
\text { characteristics by the parameter }\end{array}$ \\
\hline $\begin{array}{l}\text { HFLWA operator } \\
\text { [39] }\end{array}$ & No & No & No \\
\hline $\begin{array}{l}\text { HFLWG operator } \\
\text { [39] }\end{array}$ & No & No & No \\
\hline HFL-TOPSIS [40] & No & No & No \\
\hline $\begin{array}{l}\text { HFLWBM } \\
\text { operator [62] }\end{array}$ & Yes & No & Yes \\
\hline $\begin{array}{l}\text { HFLWHM } \\
\text { operator }\end{array}$ & Yes & Yes & Yes \\
\hline $\begin{array}{l}\text { HFLWDHM } \\
\text { operator }\end{array}$ & Yes & Yes & Yes \\
\hline
\end{tabular}

HFLWDHM operator has an excellent capability to model the correlation among multi-input parameters, and it can be applied more flexibly during the process of information fusion.

As analysed and compared the abovementioned summarization points, the superiorities of the propounded approach are summarized as follows:

Firstly, the HFLS can express the assessment information more comprehensive because it combines the excellent feature of the linguistic term and the hesitant fuzzy set. The HFLS can cope with practical problems from both quantitative and qualitative perspectives.

Secondly, the comparative analysis show that the novel approach based on the WHFLHM operator thinks over the inter-relationship among multi-input arguments, which can further reduce the loss of evaluation information and enable the decision process more flexible and valid.

Thirdly, the HFLWHM operator can be applied in different situations by assigning preference values with different parameters $\xi$, which can be seen as different risk preference by decision makers based on processing practical problems.

Because of the complicacy of the decision environment, decision makers tend to estimate the alternatives according to their preference in linguistic terms and may hesitate to determine the degree of membership. At the same time, in order to conduct a more reasonable decision in actual problems, it is indispensable for decision makers 
to concern the inter-relation of the multiple input argument. Accordingly, the propounded approach can be regarded as an effective technique for addressing practical issues.

\section{Conclusion}

In this article, by taking into account the merits of the linguistic term set and the HM operator, we extend the HM operator to HFL setting to present several HFL aggregation operators for dealing with MADM problems effectively. To begin with, we present the HFLHM operator, HFLWHM operator, HFLDHM operator, and HFLWDHM operator. Thereafter, we develop a novel MADM algorithm based on the presented operators. Ultimately, the proposed algorithm is applied to solve the practical problem, and a comparative analysis is preformed through comparing it with other MADM approaches. The most remarkable feature of the novel method is that it fully considers the correlation among multiple attributes. In addition, the proposed method has flexibility because of the adjustable parameter in the HM operator. As the parameter value changes, different ranking results of alternatives will be obtained in tackling MADM problems, which can be viewed as the risk preference of the decision makers. Furthermore, the hesitant fuzzy linguistic set can portray the incomplete, vague, and uncertain information more valid through combining the linguistic term set and the hesitant fuzzy set. In future research works, the developed aggregation operators can be utilized for other actual applications. What is more, considering the merits of the HM operator, we can generalize it to other fuzzy contexts, for instance, unbalanced linguistic term sets [59], hesitant pythagorean fuzzy set [63], and q-rung orthopair fuzzy linguistic set [64].

\section{Data Availability}

The data used to support the findings of this research are included in the article.

\section{Conflicts of Interest}

The authors declare that they have no conflicts of interest.

\section{Acknowledgments}

This work was partially supported by the National Natural Science Foundation of China (Grant no. 61372187), the Scientific and Technological Project of Sichuan Province (no. 2019YFG0100), the Sichuan Province Youth Science and Technology Innovation Team (no. 2019JDTD0015), the Application Basic Research Plan Project of Sichuan Province (no. 2017JY0199), the Scientific Research Project of the Department of Education of Sichuan Province (nos. 18ZA0273 and 15TD0027), the Innovative Research Team of Neijiang Normal University (no. 18TD08), and the Xi Hua Cup University Students Innovation and Entrepreneurship Project (no. 2019134).

\section{References}

[1] F. Xiao and W. Ding, "Divergence measure of Pythagorean fuzzy sets and its application in medical diagnosis," Applied Soft Computing, vol. 79, pp. 254-267, 2019.

[2] H. Han and S. Trimi, "A fuzzy TOPSIS method for performance evaluation of reverse logistics in social commerce platforms," Expert Systems with Applications, vol. 103, pp. 133-145, 2018.

[3] F. Shen, X. Ma, Z. Li, Z. Xu, and D. Cai, "An extended intuitionistic fuzzy TOPSIS method based on a new distance measure with an application to credit risk evaluation," Information Sciences, vol. 428, pp. 105-119, 2018.

[4] X. Peng and H. Garg, "Multiparametric similarity measures on Pythagorean fuzzy sets with applications to pattern recognition," Applied Intelligence, vol. 49, no. 12, pp. 4058-4096, 2019.

[5] P. A. Ejegwa and J. A. Awolola, "Novel distance measures for Pythagorean fuzzy sets with applications to pattern recognition problems," Granular Computing, vol. 1, pp. 1-9, 2019.

[6] C. Wei, Z. Ren, and R. M. Rodríguez, "A hesitant fuzzy linguistic TODIM method based on a score function," International Journal of Computational Intelligence Systems, vol. 8, no. 4, pp. 701-712, 2015.

[7] R. Sun, J. Hu, and X. Chen, "Novel single-valued neutrosophic decision-making approaches based on prospect theory and their applications in physician selection," Soft Computing, vol. 23, no. 1, pp. 211-225, 2019.

[8] H. Liao, L. Jiang, B. Lev, and H. Fujita, "Novel operations of PLTSs based on the disparity degrees of linguistic terms and their use in designing the probabilistic linguistic ELECTRE III method," Applied Soft Computing, vol. 80, pp. 450-464, 2019.

[9] Y. Liu, J. Liu, and Y. Qin, "Pythagorean fuzzy linguistic Muirhead mean operators and their applications to multiattribute decision-making," International Journal of Intelligent Systems, vol. 35, no. 2, pp. 300-332, 2020.

[10] L. A. Zadeh, "Fuzzy sets," Information and Control, vol. 8, no. 3, pp. 338-353, 1965.

[11] K. T. Atanassov, "Intuitionistic fuzzy sets," Fuzzy Sets and Systems, vol. 20, no. 1, pp. 87-96, 1986.

[12] K. Atanassov and G. Gargov, "Interval-valued intuitionistic fuzzy sets," Fuzzy Sets and Systems, vol. 31, no. 3, pp. 343-349, 1989.

[13] R. R. Yager, "Pythagorean membership grades in multicriteria decision making," IEEE Transactions on Fuzzy Systems, vol. 22, no. 4, pp. 958-965, 2014.

[14] R. R. Yager, "Generalized orthopair fuzzy sets," IEEE Transactions on Fuzzy Systems, vol. 25, no. 5, pp. 1222-1230, 2017.

[15] F. Smarandache, "Neutrosophic set-a generalization of the intuitionistic fuzzy set," International Journal of Pure and Applied Mathematics, vol. 24, no. 3, p. 287, 2005.

[16] Y. Qin, Y. Liu, and Z. Hong, "Multicriteria decision making method based on generalized Pythagorean fuzzy ordered weighted distance measures," Journal of Intelligent \& Fuzzy Systems, vol. 33, no. 6, pp. 3665-3675, 2017.

[17] Y. Liu, Y. Qin, and Y. Han, "Multiple criteria decision making with probabilities in interval-valued Pythagorean fuzzy setting," International Journal of Fuzzy Systems, vol. 20, no. 2, pp. 558-571, 2018.

[18] Y. Liu, J. Liu, and Y. Qin, "Dynamic intuitionistic fuzzy multiattribute decision making based on evidential reasoning and MDIFWG operator," Journal of Intelligent \& Fuzzy Systems, vol. 36, no. 6, pp. 5973-5987, 2019. 
[19] H. Garg, "Generalised Pythagorean fuzzy geometric interactive aggregation operators using Einstein operations and their application to decision making," Journal of Experimental \& Theoretical Artificial Intelligence, vol. 30, no. 6, pp. 763-794, 2018.

[20] H. Garg, "New exponential operational laws and their aggregation operators for interval-valued Pythagorean fuzzy multicriteria decision-making," International Journal of Intelligent Systems, vol. 33, no. 3, pp. 653-683, 2018.

[21] P. Liu and W. Liu, "Scaled prioritized operators based on the linguistic intuitionistic fuzzy numbers and their applications to multi-attribute decision making," International Journal of Fuzzy Systems, vol. 20, no. 5, pp. 1539-1550, 2018.

[22] P. Liu and W. Liu, "Multiple-attribute group decision-making based on power Bonferroni operators of linguistic q-rung orthopair fuzzy numbers," International Journal of Intelligent Systems, vol. 34, no. 4, pp. 652-689, 2019.

[23] V. Torra and Y. Narukawa, "On hesitant fuzzy sets and decision," in Proceedings of the 2009 IEEE International Conference on Fuzzy Systems, pp. 1378-1382, IEEE, Jeju Island, South Korea, August 2009.

[24] V. Torra, "Hesitant fuzzy sets," International Journal of Intelligent Systems, vol. 25, no. 6, pp. 529-539, 2010.

[25] Z. Pei and L. Yi, "A note on operations of hesitant fuzzy sets," International Journal of Computational Intelligence Systems, vol. 8, no. 2, pp. 226-239, 2015.

[26] H. Liao and $\mathrm{Z}$. Xu, "Subtraction and division operations over hesitant fuzzy sets," Journal of Intelligent \& Fuzzy Systems, vol. 27, no. 1, pp. 65-72, 2014.

[27] Z. Xu and M. Xia, "Hesitant fuzzy entropy and cross-entropy and their use in multiattribute decision-making," International Journal of Intelligent Systems, vol. 27, no. 9, pp. 799822, 2012.

[28] P. Quirós, P. Alonso, H. Bustince, I. Díaz, and S. Montes, “An entropy measure definition for finite interval-valued hesitant fuzzy sets," Knowledge-Based Systems, vol. 84, pp. 121-133, 2015.

[29] Z. Xu and M. Xia, "Distance and similarity measures for hesitant fuzzy sets," Information Sciences, vol. 181, no. 11, pp. 2128-2138, 2011.

[30] B. Farhadinia, "Information measures for hesitant fuzzy sets and interval-valued hesitant fuzzy sets," Information Sciences, vol. 240, pp. 129-144, 2013.

[31] Y. Liu, J. Liu, and Z. Hong, "A multiple attribute decision making approach based on new similarity measures of interval-valued hesitant fuzzy sets," International Journal of Computational Intelligence Systems, vol. 11, no. 1, pp. 15-32, 2018.

[32] N. Zhang and G. Wei, "Extension of VIKOR method for decision making problem based on hesitant fuzzy set," Applied Mathematical Modelling, vol. 37, no. 7, pp. 4938-4947, 2013.

[33] X. Zhang and Z. Xu, "The TODIM analysis approach based on novel measured functions under hesitant fuzzy environment," Knowledge-Based Systems, vol. 61, pp. 48-58, 2014.

[34] N. Chen and Z. Xu, "Hesitant fuzzy ELECTRE II approach: a new way to handle multi-criteria decision making problems," Information Sciences, vol. 292, pp. 175-197, 2015.

[35] M. Xia and $\mathrm{Z}$. Xu, "Hesitant fuzzy information aggregation in decision making," International Journal of Approximate Reasoning, vol. 52, no. 3, pp. 395-407, 2011.

[36] Y. He, Z. He, G. Wang, and H. Chen, "Hesitant fuzzy power Bonferroni means and their application to multiple attribute decision making," IEEE Transactions on Fuzzy Systems, vol. 23, no. 5, pp. 1655-1668, 2015.

[37] Z. Hong, Y. Rong, Y. Qin, and Y. Liu, "Hesitant fuzzy dual Muirhead mean operators and its application to multiple attribute decision making," Journal of Intelligent \& Fuzzy Systems, vol. 35, no. 2, pp. 2161-2172, 2018.

[38] R. M. Rodriguez, L. Martinez, and F. Herrera, "Hesitant fuzzy linguistic term sets for decision making," IEEE Transactions on Fuzzy Systems, vol. 20, no. 1, pp. 109-119, 2011.

[39] R. Lin, X. Zhao, H. Wang, and G. Wei, "Hesitant fuzzy linguistic aggregation operators and their application to multiple attribute decision making," Journal of Intelligent \& Fuzzy Systems, vol. 27, no. 1, pp. 49-63, 2014.

[40] J.-q. Wang, J.-t. Wu, J. Wang, H.-y. Zhang, and X.-h. Chen, "Multi-criteria decision-making methods based on the Hausdorff distance of hesitant fuzzy linguistic numbers," Soft Computing, vol. 20, no. 4, pp. 1621-1633, 2016.

[41] X. Gou, Z. Xu, and H. Liao, "Multiple criteria decision making based on Bonferroni means with hesitant fuzzy linguistic information," Soft Computing, vol. 21, no. 21, pp. 6515-6529, 2017.

[42] M. Grabisch, J. L. Marichal, R. Mesiar, and E. Pap, Aggregation Functions, Vol. 127, Cambridge University Press, Cambridge, UK, 2009.

[43] M. Grabisch, J.-L. Marichal, R. Mesiar, and E. Pap, "Aggregation functions: means," Information Sciences, vol. 181, no. 1, pp. 1-22, 2011.

[44] E. P. Klement, R. Mesiar, and E. Pap, Triangular Norms, Springer, Berlin, Germany, 2000.

[45] E. P. Klement, R. Mesiar, and E. Pap, "Problems on triangular norms and related operators," Fuzzy Sets and Systems, vol. 145, no. 3, pp. 471-479, 2004.

[46] E. P. Klement, R. Mesiar, and E. Pap, "Archimax copulas and invariance under transformations," Comptes Rendus Mathematique, vol. 340, no. 10, pp. 755-758, 2005.

[47] G. Beliakov, H. Bustince, D. P. Goswami, U. K. Mukherjee, and N. R. Pal, "On averaging operators for Atanassov's intuitionistic fuzzy sets," Information Sciences, vol. 181, no. 6, pp. 1116-1124, 2011.

[48] M. Xia, Z. Xu, and N. Chen, "Some hesitant fuzzy aggregation operators with their application in group decision making," Group Decision and Negotiation, vol. 22, no. 2, pp. 259-279, 2013.

[49] S. Broumi and F. Smarandache, "Single valued neutrosophic trapezoid linguistic aggregation operators based multi-attribute decision making," Bulletin of Pure \& Applied SciencesMathematics and Statistics, vol. 33e, no. 2, p. 135, 2014.

[50] D.-F. Li, "Multiattribute decision making method based on generalized OWA operators with intuitionistic fuzzy sets," Expert Systems with Applications, vol. 37, no. 12, pp. 86738678, 2010.

[51] D.-F. Li, L.-L. Wang, and G.-H. Chen, "Group decision making methodology based on the Atanassov's intuitionistic fuzzy set generalized OWA operator," International Journal of Uncertainty, Fuzziness and Knowledge-Based Systems, vol. 18, no. 6, pp. 801-817, 2010.

[52] G. Wei, R. Wang, J. Wang, C. Wei, and Y. Zhang, "Methods for evaluating the technological innovation capability for the high-tech enterprises with generalized interval neutrosophic number Bonferroni mean operators," IEEE Access, vol. 7, pp. 86473-86492, 2019.

[53] P. Liu and X. Zhang, "Some intuitionistic uncertain linguistic Bonferroni mean operators and their application to group decision making," Soft Computing, vol. 23, no. 11, pp. 38693886, 2019. 
[54] T. Hara, M. Uchiyama, and S.-E. Takahasi, "A refinement of various mean inequalities," Journal of Inequalities and Applications, vol. 1998, no. 4, Article ID 932025, 1998.

[55] J. Qin, "Interval type-2 fuzzy Hamy mean operators and their application in multiple criteria decision making," Granular Computing, vol. 2, no. 4, pp. 249-269, 2017.

[56] S. Wu, J. Wang, G. Wei, and Y. Wei, "Research on construction engineering project risk assessment with some 2tuple linguistic neutrosophic Hamy mean operators," Sustainability, vol. 10, no. 5, p. 1536, 2018.

[57] P. Liu and X. Liu, "Linguistic intuitionistic fuzzy Hamy mean operators and their application to multiple-attribute group decision making," IEEE Access, vol. 7, pp. 127728-127744, 2019.

[58] J. Wang, G. Wei, J. Lu et al., "Some q-rung orthopair fuzzy Hamy mean operators in multiple attribute decision-making and their application to enterprise resource planning systems selection," International Journal of Intelligent Systems, vol. 34, no. 10, pp. 2429-2458, 2019.

[59] F. Herrera, E. Herrera-Viedma, and L. Martinez, "A fuzzy linguistic methodology to deal with unbalanced linguistic term sets," IEEE Transactions on Fuzzy Systems, vol. 16, no. 2, pp. 354-370, 2008.

[60] Z. Xu, "Intuitionistic fuzzy aggregation operators," IEEE Transactions on Fuzzy Systems, vol. 15, no. 6, pp. 1179-1187, 2007.

[61] Z. Xu and R. R. Yager, "Some geometric aggregation operators based on intuitionistic fuzzy sets," International Journal of General Systems, vol. 35, no. 4, pp. 417-433, 2006.

[62] D.-N. Liu, "Model for evaluating the electrical power system safety with hesitant fuzzy linguistic information," Journal of Intelligent \& Fuzzy Systems, vol. 29, no. 2, pp. 725-730, 2015.

[63] H. Garg, "Hesitant Pythagorean fuzzy Maclaurin symmetric mean operators and its applications to multiattribute decision-making process," International Journal of Intelligent Systems, vol. 34, no. 4, pp. 601-626, 2019.

[64] H. Wang, Y. Ju, and P. Liu, "Multi-attribute group decisionmaking methods based on q-rung orthopair fuzzy linguistic sets," International Journal of Intelligent Systems, vol. 34, no. 6, pp. 1129-1157, 2019. 